\title{
Determination of effective temperatures for an extended sample of dwarfs and subdwarfs (F0-K5)
}

\author{
A. Alonso, S. Arribas and C. Martínez-Roger \\ Instituto de Astrofísica de Canarias, E-38200 La Laguna (Tenerife), Spain \\ Electronic mail: aas@ll.iac.es, sam@ll.iac.es and cmr@ll.iac.es \\ Received May 22; accepted July 27, 1995
}

\begin{abstract}
We have applied the InfraRed Flux Method (IRFM) to a sample of 475 dwarfs and subdwarfs in order to derive their effective temperatures with a mean accuracy of about $1.5 \%$. We have used the new homogeneous grid of theoretical model atmosphere flux distributions developed by Kurucz $(1991,1993)$ for the application of the IRFM. The atmospheric parameters of the stars cover, roughly, the ranges: $3500 \mathrm{~K} \leq T_{\text {eff }} \leq 8000 \mathrm{~K} ;-3.5 \leq[\mathrm{Fe} / \mathrm{H}]$ $\leq+0.5 ; 3.5 \leq \log (g) \leq 5$. The monocromatic infrared fluxes at the continuum, and the bolometric fluxes are derived using recent results, which satisfy the accuracy requeriments of the work. Photometric calibrations have been revised and applied to estimate metallicities, although direct spectroscopic determinations were preferred when available. The adopted infrared absolute flux calibration, based on direct optical measurements of angular stellar diameters, sets the effective temperatures determined using the IRFM on the same scale than those obtained by direct methods. We derive three temperatures, $T_{J}, T_{H}$ and $T_{K}$, for each star using the monochromatic fluxes at different infrared wavelengths in the photometric bands $J, H$, and $K$. They show good consistency over $4000 \mathrm{~K}$, and no trend with wavelength may be appreciated. We provide a detailed description of the steps followed for the application of the IRFM, as well as the sources of the errors associated to the different inputs of the method, and their transmission into the final temperatures. We also provide comparison with previous works.
\end{abstract}

Key words: stars: fundamental parameters — stars: Population II — stars: subdwarfs — stars: general

\section{Introduction}

The stellar effective temperatures might be, in principle, determined following a fundamental or direct method based on the combination of the bolometric fluxes and the angular diameter measurements according to the equation:

$$
T_{\text {eff }}=\left(\frac{4}{\sigma}\right)^{1 / 4} \theta^{-1 / 2} F_{\text {Bol }}^{1 / 4},
$$

where $\sigma$ is the Stefan-Boltzmann constant, $\theta$ is the angular diameter of the star, and $F_{\mathrm{Bol}}$ is the bolometric flux measured on the surface of the earth.

However, in practice, we need atmosphere models to perform secondary corrections to both the angular diameter measurement (limb darkening) and $F_{\text {Bol }}$ (interstellar absorption). The Sun is obviously the only exception, since its limb darkening can be empirically determined, and its interstellar absorption is negligible. In any event, the main difficulty affecting the direct procedure is the limited number of stars with a reliable measurement of their angular

Send offprint requests to: A. Alonso

* Table 4 is only available in electronic form at CDS Tables 1-3 are also available via ftp 130.79.128.5 diameter (see for instance Table 4 from Mozurkewich et al. 1991). This problem is remarkably severe for the low main sequence, as there are no direct measurements of angular diameter for stars later than F5V, with the above mentioned exception of the Sun. For this reason, relatively large uncertainties still remain on the scale of stellar effective temperatures in the low main sequence, especially when the effect of metallicity is considered.

Thus, we are compelled to use semi-direct methods, which require, as a basis, atmosphere models in addition to observational information (see the review by BöhmVitense 1981). Among the different methods of this type the Infrared Flux Method (hereafter IRFM; Blackwell et al. 1990, and references therein) seems especially adequate to analyse the temperatures of F, G, and $\mathrm{K}$ stars. The IRFM has been successfully applied to different samples of population I stars (e.g. Blackwell et al. 1990; Bell \& Gustafsson 1989; Saxner \& Hammarbäck 1985). However, the works based on the IRFM devoted to metal poor stars (Magain 1987; Arribas \& Martínez-Roger 1987, 1989) are restricted to rather limited samples.

The present paper is part of a long term programme aimed to a better definition of the scale of temperatures 
Table 1. The calibration of $q$ - and $R$ - factors versus metallicity, $\log (g)$ and effective temperature in $\lambda_{\text {eff }}=1272.5 \mathrm{~nm}($ band $J)$, computed using models by Kurucz $(1991,1993)$. The units of $R$-factors are (nm)

\begin{tabular}{|c|c|c|c|c|c|c|c|c|c|c|c|c|c|}
\hline & & \multicolumn{2}{|c|}{$[P 0 / 1]=0,0$} & \multicolumn{2}{|c|}{ 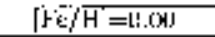 } & \multicolumn{2}{|c|}{ Fri' } & \multicolumn{2}{|c|}{$[\Gamma r / T T]=-2-i$} & \multicolumn{2}{|c|}{$[I, y]]=-9,0$} & \multicolumn{2}{|c|}{$\left[H_{i, j}, H^{\prime}\right]=-\bar{j} . \bar{i}$} \\
\hline inei & $19(0)$ & (1) & I & $71.5 \dot{\mid}$ & ia.ni & $\pi]$ & hoplitir] & $g[2]$ & $\log \left[d \mathrm{de}^{\circ}\right.$ & $z_{2}^{\prime}[\sqrt{1}]$ & $\lg [f]$ & $r_{i}^{\prime}, J^{\top}$ & أ \\
\hline 5.5110 & 4 & 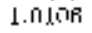 & 7. & 1.0114 & 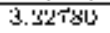 & 1.17717 & \pm 25010 & l.tutise & 5 -ijlint4 & $1.611 \mathrm{l}$ & 7819 & & - \\
\hline زן & 5. & 1.0150 & 320760 & $1.012 \mathrm{t}$ & $729+4$ & $1 . \tan 13$ & . 2.7101 & 1.!Ki:! 7 & $\therefore .24574$ & - & & - & - \\
\hline RTEO & A.110 & 1.011\% & 122550 & 1.0104 & $7,2,350$ & ثان & 3. & 1.0107 & 3.25:24 5 & רוו ריוּו. 1 & 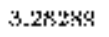 & I.RInI & 3.28121 \\
\hline ' & 5.40 & 1.117 .38 & ] & 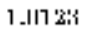 & ㄱ. 213010 & $1.11 \perp .5$ & $72 \div 48$ & 1,1010; & $5.281+y$ & -- & - & - & - \\
\hline tollo & 1.110 & 1.117 .37 & :1.2H154 & $1.111+14$ & 7.2445 & 1,600 & 3.30197 & 1, 0001 & 3.3545 & 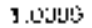 & 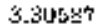 & נווונימ. & 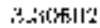 \\
\hline 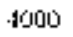 & 50 & $1.117 \$ 1$ & 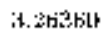 & 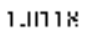 & 장 & 1,1112 & 1.2904 & 1,10103 & $3.30+1.5$ & - & . & . & - \\
\hline 4-45ו & t.Lio & 1. 1714 & 2. 24132 & ה ה ועם 1 & A.51007 & I . MU:A & 7728 & 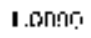 & $3.320 \mathrm{Gt}$ & 1,007: & $5-9313$ & 9001) & $330 \mathrm{~N}$ \\
\hline $42: 3$ & 5. & 1 LLIJ 13 & 3. & ה 1 רוע. L & 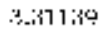 & לםا1וة. 1 & ! & 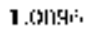 & 371015 & 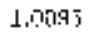 & Titlityitis & & \\
\hline 4E[1"- & تالة. 1. & $1.0 \mathrm{~J} B 3$ & 3.3207 & L.U1'? & 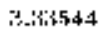 & 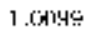 & 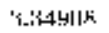 & 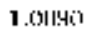 & 3.7519 & 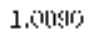 & 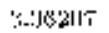 & [ I & 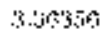 \\
\hline 450100 & 5,00 & 10154 & 3.32179 & 1.0124 & 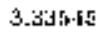 & 1.나년 & B_twit & 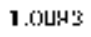 & Zi.it5its: & 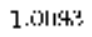 & 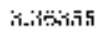 & 1 이애논 & 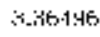 \\
\hline $4 \bar{i}=0$ & 4.110 & I_ח!fil & ר. & 上. 1134 & 3.39348 & 自 & $3.3 \overline{7006}$ & 1,0018 & 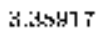 & 1. & 3.StrEO & 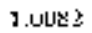 & זי1ישיع_ה \\
\hline $47=10$ & 5. .110 & I.IIIR4 & 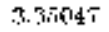 & 1,10131 & 3.9835 & 1.0100 & 3.37915 & 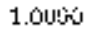 & 3.45454 & 1.닞 & 3.145 .51 & ]. OLUE & $3.3 \mathrm{~s}$ \\
\hline 政140 & 1. .110 & $1 . \mid 17 \mathrm{Kis}$ & 618 & דיה: & :3.379744 & $1,01 \mathrm{n}$ & 3.41155 & 1.0010 & 0.13286 & 1,000 & 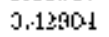 & $1,100101]$ & 3.5000 \\
\hline قِالة & 5.110 & $1.117 \%$ & astors & 1.CI1:HT & $\therefore 37440$ & 1,0101 & $3.4104 \bar{i}$ & 1.00is & 3.42215 & 1.0085 & $3,4 \$ 502$ & 1, & 3.6000 \\
\hline 1050 & 1.00 & & $3.172+1$ & ב & 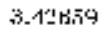 & 1.011:t+t & 'H.44H7 & 2.نثاו 1 & 7.45869 & דירוחואו & 6.49485 & 1, & T.4GRIA \\
\hline 5250 & 5.00 & ני. & $3.172 \mathrm{z}$ & 10 & S.4:6Ac & $7.011:+5$ & 'i.4.44.41 & לי'יناו & :i.45T47 & إיצוחוֹ & 9.4742 & 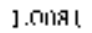 & $9.46 \mathrm{int}$ \\
\hline FFilition & 4,100 & בגו & 344018 & 1. 1. & S.joluts & ]. & 3.tstst & ].0ugl & 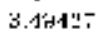 & \$ & 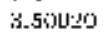 & ד' דים. ] & 3.50141 \\
\hline & 0,00 & 1.0173 & $3.4+4+7$ & 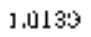 & 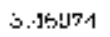 & 1.ousy & ילטים & 1.0uरs & З.4मth & ודידנווי..ר & 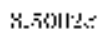 & ר. & 1.50l \\
\hline$\therefore 75 i$ & +. liäi & I. & .74525 & 1,0191 & $3.4905 \%$ & 1.0097 & $3 . ; 1 \%$ & 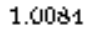 & 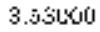 & إنتإلأ. & 3.3513 & 1 & 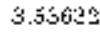 \\
\hline 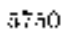 & 5. Llio & ה. ה.וI1 & $5+4130$ & 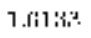 & 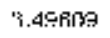 & 1,0196 & $3 . \overline{1} 1856$ & 1,01014 & 9.53509 & $1.00 \%$ & $3,5=95$ & i, & 5.5714 \\
\hline 6الثا & 1.100 & 1 I.IIf & 3.57596 & 104144 & $\therefore \sin$ & 1,0000 & 5.5544 & $1.000 \%$ & ו & 1.0003 & 3,3005 & 1,0002 & 3404 \\
\hline 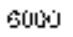 & 5.110 & 1515. & & 1:141:14 & 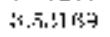 & ד' & A.s.tidil & 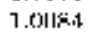 & B. & 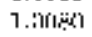 & 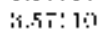 & 15969 & ' \\
\hline 1950 & 1.100 & 1.156 & 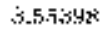 & 1:1ו1. 7 & 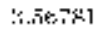 & 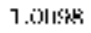 & $\therefore . \overline{1} 484$ & 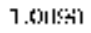 & 4. & ה & '. & 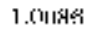 & ' \\
\hline ודיהיהצי9 & $\therefore, 00$ & 1.0151 & 3.56 .540 & 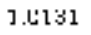 & 3.5tist & i & 3.5.5.5. & 1. & גיורונה ני & 1.|ง & 1. & 30 & 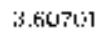 \\
\hline olsos & 4.100 & I.160 & 3 & 1. & - Fionitser & דילקlli. & A_teryis & ביצוاום. & 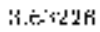 & ו'بتיباוا'.1 & 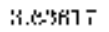 & لـ: & i.H.'xis \\
\hline Iים & 5,00 & 1.DI1.1 & 338012 & J.LLI & Stost' & 1.6ues & 3.tstst & 7.0108 & B.6stu & 7.USE & B.ETUา & ז' ז' & 3.6. \\
\hline וֹ & 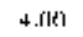 & 1.1141 & .762578 & $1, \mathrm{C} \perp 11$ & 3.386 & 1.0085 & 3. & 1. CIÖا & 3.0000 & 1. & 3.4650 & 1,0003 & 3.600 .6 \\
\hline 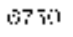 & $\overline{01}, 1,010$ & $1, B 193$ & $3.1024 \% 1$ & 1.0106 & 3.3.317 & (6083 & $3.0 \mathrm{btg} \mathrm{0}$ & ل' 1. 1. & 3.304 & 1. & 3.483rs & ק ד' & 3.6-1.5 \\
\hline ائيةiآ & $\dot{4} .1 \mathrm{i} i \mathrm{I}$ & 1:1920 & 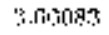 & 1, $\Gamma \perp \cap \mathrm{NB}$ & 3,$0 ; 310$ & $1,00 \% 0$ & 9,6013 & בדידסטס & & 1. & 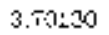 & $1,00 \pi 3$ & 3.702040 \\
\hline וחו"ה & 1. & 1. & 3.69015 & $1, r, 000$ & 3.: & 1.0073 & 3.09398 & 1. & 3. rijacou & 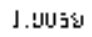 & 3.roges & 1.60.is & 3.54540 \\
\hline T2:1: & ום & ( $.612,5$ & 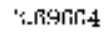 & 1,5095 & 3,50811 & 1,0009 & 0,53049 & 1.00:5:5 & 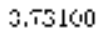 & ; & 3,T5] & 1,0000 & 9,35121 \\
\hline 125í & 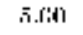 & ו. & 3.109541 & $1,00 \% 3$ & 3.70015 & 1.0001 & 3,5300 & 1. & 3.7.675 & ] [ [III:] 1 & 3.7.399a & $1.00 \cdot 17$ & 3.T:301 \\
\hline Tison & 4.61 & 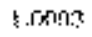 & 5.7444 & $1,5 i] 1,5$ & 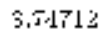 & $1,10.50$ & 3.513 & 1,01017 & 3.8113 & 1. & $3, T 60 \bar{i}$ & 1,9048 & 3,70010 \\
\hline TER & (1, I, & 1, Dofs & 3.73010 & 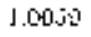 & 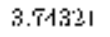 & 1.0037 & 3.7003 & 1. & 3.Totsh & I. [IU 3 3? & 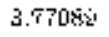 & J $600 \mathrm{~B}$ & 3.T: \\
\hline$T 7=1$ & 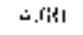 & 1. 12172 & :P.tings & 1,0041 & 3.T.5.7 & 1,0022 & $0,50 \mathrm{ag}$ & 1,0013 & 3.20 .15 & 1.0010 & & 1,0010 & 3.80725 \\
\hline TT & 今.CM & 4., 1, 1 & 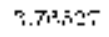 & 1.5:125 & $3,5 \div \div 4 !$ & $1,001.8$ & 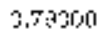 & 1,0000 & $3.5 \mathrm{gigh}$ & $1.001-1$ & $3,9024 \bar{i}$ & 1,101019 & 3.9024 \\
\hline ודיה & $\leq \mathrm{BO}$ & 1.198 & 3.6010 & 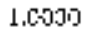 & 3.81700 & $1.000 \mathrm{~s}$ & 3.85178 & $1.000 \mathrm{M}$ & 3.83565 & 1.A0124 & 3.92083 & 1.0001. & 3.840 .44 \\
\hline 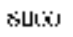 & $\therefore$ & U. \$ & 'i.:301t?7 & 7. & 3.911 .5 & 1,0000 & 3.325 .50 & 0.9019 & 3.9315 & 1.0000 & 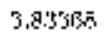 & 0,9495 & 3.83479 \\
\hline$\$ 2.7$ & (E) & $1.60+6$ & :is427T & 1.Mol & 3.8535 & 0.6004 & A.4ini: & $0, \$ 001$ & $3.8 \mathrm{i}$ & 0.6ngel & 3.9- & 0,50012 & .8 .87400 \\
\hline$\$ 21$ & 5.6 & 0. Sulti $_{\mathbf{B}}$ & $\therefore$ ind, & נ1 & $3.8 .5 \perp 1.3$ & 0.9049 & :1.4A5, & ח, 90,12 & 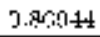 & B.90दs & 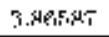 & 11.5074 & ד.sent: \\
\hline
\end{tabular}

for $\mathrm{F}, \mathrm{G}$, and $\mathrm{K}$ dwarfs and subdwarfs, using the IRFM. This work is relevant to three main topics: (a) analysis of the global behaviour of atmosphere models (e.g., Magain 1987), (b) the correct interpretation of the observed HR diagram (e.g., Arribas \& Martínez-Roger 1988, 1989), and (c) fine spectroscopic analysis for abundance determinations of metal poor stars (e.g., King 1994).

The general programme has been focussed on the improvement of the different factors which affect the accuracy in the definition of the temperature scale. Firstly, the sample of selected stars has been substantially enlarged compared to previous works (up to almost 500 stars). This point is particularly important in order to properly sample wide ranges in colour and metallicity. The accurate infrared photometry required for the application of the IRFM was measured for $75 \%$ of the stars in the sample (Alonso et al. 1994a, Paper I). Secondly, due to the sensitivity of the IRFM temperatures to the infrared absolute flux calibration considered, this subject was revised in Alonso et al. (1994b, Paper II). There, a new absolute calibration for the infrared flux of Vega, which scales the IRFM temperatures to those derived by direct methods, is proposed. Thirdly, a method to obtain bolometric fluxes for metal poor stars was devised in Alonso et al. (1995, Paper III). Last, but not least, we have used the improved grid of atmosphere models computed recently by Kurucz (1991, 1993).

This paper provides detailed information on the procedure followed to derive the effective temperatures for the whole sample. The paper is laid out as follows. Section 2 explains the practical implementation of the IRFM. The theoretical and observational inputs of the method, as applied here, have been separated in order to discuss the influence of errors in the derived temperatures. Thus, Sect. 3 analyses the theoretical $R$ - and $q$-factors, and the observational inputs are discussed in Sect. 4. This section also includes a description of the criteria adopted to collect the sample of stars, a revision of photometric calibrations to estimate the metallicity, and the correction of interstellar extinction. The effective temperatures are derived 
Table 2. The same that Table 1 for $\lambda_{\text {eff }}=1635.0 \mathrm{~nm}$ (band $H$ )

\begin{tabular}{|c|c|c|c|c|c|c|c|c|c|c|c|c|c|}
\hline \multirow[b]{2}{*}{$i_{e j}$} & \multirow[b]{2}{*}{$\operatorname{tsg}(\theta)$} & \multicolumn{2}{|c|}{$\Gamma e / T T=0.510$} & \multicolumn{2}{|c|}{$\left[\Gamma_{n} / \mathrm{II}-\overline{0}, \overline{0}\right.$} & \multicolumn{2}{|c|}{$\neg \mathrm{F}_{m} \mathrm{H} \mid=-\mathrm{I} . \mathrm{b}$} & \multicolumn{2}{|c|}{$.|\mathrm{F}, \mathrm{H}|=-2.4$} & \multicolumn{2}{|c|}{ 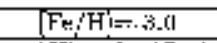 } & \multicolumn{2}{|c|}{$\Gamma 0 / \mathrm{TI}=-3, j$} \\
\hline & & 至 & $\operatorname{Lg}\left[H_{S}\right)$ & $\overrightarrow{\nabla i n j}$ & l'm & $a[\pi]$ & $i_{i j}\left[R_{n} j\right.$ & (1) & 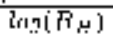 & $\pi[I]$ & agdTri & (1) & $i \omega \mid R_{h} h$ \\
\hline 550 & 1.40 & $7.425 \overline{1}$ & $\therefore 2 \%+41$ & 10.6\% & $\overline{7,201913}$ & 1,0572 & 901000 & 1,05istis & 3.417 .913 & 1.0 .522 & $336 \%$ & - & - \\
\hline$\$ 40$ & 5.400 & $7.11=\overline{1} \bar{i}$ & X313045 & $1,05.50$ & 0,3343 & $1,01,52$ & 30505 & $1,05,102$ & 3.10015 & - & - & - & - \\
\hline 30 & 1.40 & 1.INTS & 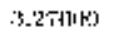 & 7.0its & 7. 5 5ार19 & 1.0579 & 8.98172 & 1.5490 & 9.4115 .3 & $1.0 \pm 44$ & $54100 \pi$ & $5,0+19$ & 34255 \\
\hline 3750 & 5.00 & 1.0612 & 3 SกT & $3.0=24$ & 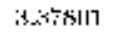 & $1.1143 \mathrm{sin}$ & 3.40675 & 7.04 ins & 4.41396 & - & - & - & - \\
\hline 1010 & 1.00 & 1.0725 & 30564 & ] & A) & $1 .[150$ & 3.20405 & $3.048 t$ & $5.44: 112$ & $1.07 \bar{i}]$ & :-4.तng? & T. $05,1+4$ & $8.48=111 !$ \\
\hline 4000 & 50 & 1.0037 & 3000 & ].05s & 3.1140 .3 & 1.UA5! & 3. & 3. & $\therefore .4620 \%$ & - & - & - & - \\
\hline 4250 & 1.00 & 1.0603 & $3.40+13$ & $1.06 \mathrm{~g}$ & 3.11531 & 1. & $3.1 \$ 010$ & 1.01 .5 & $5.4-\operatorname{Ten}$ & I. & 8 8ถิ157\% & ]. & 3.3242 \\
\hline 4250 & 5,000 & 10.599 & $3,410 \div 9$ & $1.00 \mathrm{is}$ & 3.42170 & $1.14 \pm 6$ & 3.47250 & 1.034. & 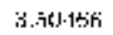 & 1. & $3.58 J^{\prime}$ & - & - \\
\hline 4000 & $\$, 00$ & 1.0500 & $3,4 \bar{i} 501$ & 1.0 .52 & $3.4724 ;$ & 1, M년 & $3.401 \% 4$ & $1.0 .11 t$ & 3.72311 & 7.4.5ist & 3.5540 & 1. & 3.56514 \\
\hline 1.501 & E. $6 \%$ & 1 . 11527 & & 1. & $7-47 \pm 00$ & 1,0405 & 0,30164 & 1.005: & & 1.0279 & $3.570 \%$ & 1.0201 & 3.5500 \\
\hline 1754 & $b_{1}(K)$ & $1.1: 426$ & : $7.645 \mathrm{~K}$ & $7 .-14: 4 \mathrm{t}$ & s.nithde & $7.0+70$ & it. 55421 & 7. AILES & 3.578070 & $1,052 a$ & $3 \sin 7$ & 1.0819 & .7. \\
\hline 17 & B..LY & 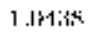 & $\therefore .538+1$ & 1 1. 1. & ; & $1,040 \overline{1}$ & 35584 & 1, (1):120 & & 1.0370 & 9,131845 & 1.0202 & $3.8302 \bar{i}$ \\
\hline 50100 & 1,104 & $1.10 \mathrm{SOS}$ & 3.54 .552 & 1 . & 3.5145 .5 & ר. & ;.5134ty & 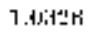 & & 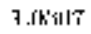 & :ETHS1 & 7. OH'then & RA18 \\
\hline 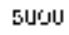 & 5.LII & רنנنا. & 3.58451 & 1 _LVSTE & 3.3AKRO & 7.6\%AL & ;51:414 & 1. & $8.3+271$ & 1.0284 & 7.64ili31 & $7.02 \mathrm{~A}^{2}$ & ИEHLT \\
\hline 0250 & 4.00 & i.05t & $3,6 \mathrm{stdt}$ & 1.0200 & 3bist 15 & $1.0 \mathrm{ars}$ & $3.71 \geq 2$ & 1. & $\operatorname{sex} 12$ & נ. ThST & $\therefore B H A B 4$ & T. & B.AAGOT \\
\hline 0250 & ה. & 1.0306 & $3 . E_{4} F \mathrm{BO}$ & L _. & 3.05010 & J ofts & 237041 & I. dust & & ].1455 & В.т人सบ & $7.015 \mathrm{Fk}$ & B.TUJift \\
\hline חחיריה & $4.6 \pi$ & I. I.525 & 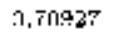 & 1.0258 & $\therefore, \because 1240$ & 1,61302 & דi 3001 & $1.02 \mathrm{GL}$ & & $1.0 \mathrm{105}$ & 3,7430 & 1.0007 & 0.74428 \\
\hline סדירוּ & $\overline{5}, 60$ & 1. & J, 70050 & 1.0244 & 3.70903 & $1,13 j 4$ & 3 isiso & 1.0230 & & $3,0 \mathrm{t} 1$ & $3, \pi-1 ! 11$ & 1.0038 & 3.75158 \\
\hline 575 & 4.6I & $1.01 \% 5$ & 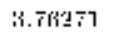 & 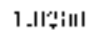 & 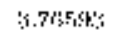 & 1. $n 2 \% 4$ & :1, - & $1.129 \overline{1}$ & $4, \bar{i} s$ & 1,0240 & 3,10111 & 1,024 & : נ. \\
\hline STTEU & .60 & โ & ה.ד & 1 ـLIIIUU & $3.70 \mathrm{TT}$ & ד'רנים. & B. & 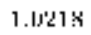 & & 7. (152) & BATARAB & 7.0205 & ห.THAา \\
\hline 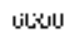 & . & 1. & $3.51 .46]$ & 1.4765 & З.าก & 7.6141 & $\therefore 8 \% 0 \$ 7$ & 1. 12001 & $\triangle A B T 5$ & 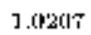 & 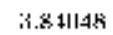 & $1.02 \mathrm{ks}$ & S.s1718 \\
\hline 1000 & 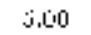 & E. & 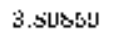 & 1.U1EU & דT 3.813 & $7.4181 \mathrm{k}$ & 3546 & $1.47: 5$ & - & 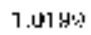 & $828+25$ & דצים & 381234 \\
\hline 0250 & . 1.00 & 1.0125 & & 1.0136 & $81 \mathrm{~s}$ & 1.0108 & بو & 1, & & 7.0171 & 3.25 & מאדנים & $3.2 \times 88$ \\
\hline 0250 & $5, r_{1}, 0$ & 2,5111 & 3,35859 & . & $3.9 \mathrm{gat}: \mathrm{i}$ & $1.0 \mathrm{~L} \mathrm{~L} 0 \mathrm{0}$ & 3.57 ids & 1.0160 & 0,89549 & 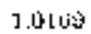 & 3,45890 & ניהי1.01 & 3.85027 \\
\hline b.j00 & - & 1'- & 34178 & 1.0108 & $3016 \mathrm{~B}$ & 1.0135 & 3 & 1. D11:t & 3.3130 & Tht ] & उ.\$BST & I.014 & 3.00130 \\
\hline Brith & 1001, & 1,rents & & $1.000 \mathrm{~A}$ & 60 & 1.0127 & 403 & 1.01 .50 & & $1,131 \div 3$ & 3,95 & 1.0145 & $0, \$ 454$ \\
\hline Fit다 & 4 & J. 애T & I & f.11084 & 5,797972 & $1 . \Pi \underline{1} 11$ & דים & $1 . \Pi 118$ & :19\%RP & ب. & 3.97895 & והבדנו. & 3.57001 \\
\hline BTE, & & $1,00 j 2$ & $0,5,-50$ & נ.0000, 1 & 3,95090 & $1,0 \mathrm{i00}$ & 300070 & 1.0110 & 0.97574 & $1, ß 1 \bar{\alpha}$ & 0.97820 & 1.0113 & 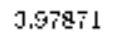 \\
\hline 701119 & II!II & 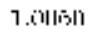 & & 1. 1.10\% & 4 & 1. ПLस:- & $16 \mathrm{Fi}$ & 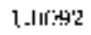 & & 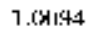 & 4.02214 & 7. & 4.6zrlos \\
\hline Fotiा & $\overline{7} .611$ & 1. & 7. & דogot, & $0,004.93$ & 10070 & & 100 & & 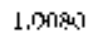 & $4,02 !$ & | & 4,0 \\
\hline 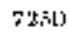 & 4 & 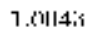 & 4.114070 & I. 11044 & ו & 1.01070 & 4.0.0001] & 1. 1 1 & i & 1.61174 & 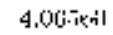 & 1.4IIIT5 & $4.01, \omega_{4}$ \\
\hline סקהמת & וاוה, 5 & 4) & 4.0 & 1. (noles & 410 & 1,0040 & 1דיהם & 101049 & & $1,0,015$ & 4,60 & $1.45 \mathrm{a}$ & $4,6045 \overline{1}$ \\
\hline T대이이 & 4.4111 & אסטווה. & & 1. & $975+4$ & L. Inos, & רसR: & 1 ו & & $1.0 \Pi=8$ & & I $U \mathrm{H}$ & 4.1176, \\
\hline 7500 & $5.111]$ & 0,9090 & 4.09471 & 0,0084 & t.rings & 1.0010 & ד & 10003 & & 1,0024 & 4.10. & 1,0025 & $4.10 .5 \% 7$ \\
\hline סה & 4.1111 & 11.5491 & 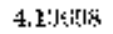 & 0.9001 & t. $1+254$ & 1 & 4 Longs & ניביזונ & 4.15 & 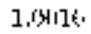 & 4.15.14: & 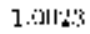 & $4.15 \%$ \\
\hline 00ה & ५.!॥। & 111945 & $4.1292 \overline{1}$ & |I..9\%קה & $4.1 \mathrm{~g}=11$ & 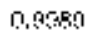 & Alit! & 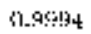 & & 7. & $4.7+40 \overline{8}$ & ب4. & $4.7465 T$ \\
\hline काוा & 4.IIII & (1. & 4. 584 & {$[1,9974$} & 840 & $1,000 \mathrm{H}$ & 4.19202 & 0.0007 & & 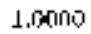 & 4.1081 .5 & 1. & $4.1[95=1$ \\
\hline סוונים & ㄷ..IIII & 4 רبي:. & 4.7745 & 11.45\% & 4. 1 idth & ים, & $4.18 ! 1 \mathrm{l}$ & 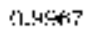 & $4.1545 \bar{i}$ & $11.4+471$ & 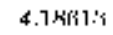 & 11.947 & $4.7 \mathrm{~s}: 114$ \\
\hline$\therefore 20$ & 4 & $0.048 \overline{1}$ & $4.27 \% 74$ & 11.997 & 4.23021 & 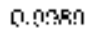 & 4.27m? & 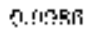 & 4. & II.SHARE & 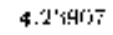 & 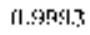 & $4.280 \mathrm{mH}$ \\
\hline $8: 00$ & 5..IJ!i & 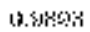 & 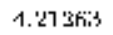 & IJ.MS: & 4. $212 \Pi 14$ & ה & 1.22467 & 1 & 4.12054 & $11 . \$ 4+1$ & 62874 & $11.9 \mathrm{HO}$ & $1.22 \pi^{4}-1$ \\
\hline
\end{tabular}

in Sect. 5, where the internal consistency of the method and the uncertainties affecting $T_{\text {eff }}$ are assesed. The final temperatures are compared to common determinations of previous works in Sect. 6. Finally, in Sect. 7, the main results are briefly summarized.

In a forthcoming paper, we will provide and discuss the calibrations $T_{\text {eff-colours- }}[\mathrm{Fe} / \mathrm{H}]$, as well as the mean intrinsic colours for dwarfs and subdwarfs.

\section{The implementation of the IRFM}

The InfraRed Flux Method (Blackwell et al. 1990) uses the quotient between the bolometric flux $\left(F_{\mathrm{Bol}}\right)$ and the monochromatic flux at a chosen infrared wavelength of the continuum $\left(F\left(\lambda_{\mathrm{IR}}\right)\right)$, both measured at the surface of the earth, as indicator of $T_{\text {eff }}$. This quotient is the so-called observational $R$-factor $\left(R_{\text {obs }}\right)$. The theoretical counterpart derived from models, $R_{\text {theo }}$, is obtained as the quotient between the integrated flux $\left(\sigma T_{\text {eff }}^{4}\right)$ and the monochromatic flux at $\lambda_{\mathrm{IR}}\left(F_{\bmod }\left(\lambda_{\mathrm{IR}}\right)\right)$, at the surface of the star. Thus the basic equation of the IRFM reads:

$$
\begin{aligned}
R_{\mathrm{obs}}=\frac{F_{\mathrm{Bol}}}{F\left(\lambda_{\mathrm{IR}}\right)}= & \frac{\sigma T_{\mathrm{eff}}^{4}}{F_{\mathrm{mod}}\left(\lambda_{\mathrm{IR}}, T_{\mathrm{eff}},[\mathrm{Fe} / \mathrm{H}], g\right)}= \\
& =R_{\mathrm{theo}}\left(\lambda_{\mathrm{IR}}, T_{\mathrm{eff}},[\mathrm{Fe} / \mathrm{H}], g\right)
\end{aligned}
$$

where the explicit dependence on metallicity, surface gravity, and $\lambda_{\mathrm{IR}}$ is taken into account. The IRFM only requires from models the correct prediction of the continuum IR fluxes (note that the bolometric flux at the stellar surface is fixed by the value of $T_{\text {eff }}$ ). This requirement seems relatively easier to fulfill, if compared to those demanded by other semi-direct methods. In particular, free-free and bound-free transitions of $\mathrm{H}^{-}$ion, the main source of the continuum opacity in the IR for F, G and $\mathrm{K}$ stars, are relatively well understood. Therefore, in principle, the dependence on models is not a critical point to the IRFM, at least for spectral types earlier than late $\mathrm{K}$ where the IR opacity due to molecular bands is of minor importance. Another advantage of the IRFM, as explained in Sect. 4.1, 
Table 3. The same that Table 1 for $\lambda_{\text {eff }}=2175.0 \mathrm{~nm}$ (band $K$ )

\begin{tabular}{|c|c|c|c|c|c|c|c|c|c|c|c|c|c|}
\hline \multirow[b]{2}{*}{ is } & \multirow[b]{2}{*}{ inis] } & \multicolumn{2}{|c|}{$\left[I_{1}: I I\right]=0,50$} & \multicolumn{2}{|c|}{$[\mathrm{Fe} / \mathrm{II}]=0.00$} & \multicolumn{2}{|c|}{$\mathrm{FH} H=-1.0$} & \multicolumn{2}{|c|}{ TFin/Hi=-3.U } & \multicolumn{2}{|c|}{$[\mathrm{Fe} / \mathrm{H}]=-i, 0$} & \multicolumn{2}{|c|}{$\left[\mathrm{F}_{n !} \cdot \mathrm{H}\right]=-i_{-5}$} \\
\hline & & $5 \mid 4 i$ & $\operatorname{lng}\left[R R_{\mathrm{K}}\right]$ & in & $k_{n}\left[\mathbb{R}_{x}\right.$ & $\pi k !$ & $m_{j}\left[\overline{n_{k}}\right)$ & $y\left(F^{\prime}\right)$ & $\operatorname{lon}(\pi, k) !$ & $r\{R\}$ & $\log [\mathrm{d} / \mathrm{m})$ & $9 \pi$ & $\operatorname{lng}(19)$ \\
\hline 1जoll & 4.대I & $1 .[145]$ & 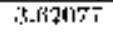 & 1.010 & 7,8040 & 1.030 & 3.71134 & j. & $3.0 \mathrm{rr}^{2} 20$ & $1.4 \div 67$ & B.7'3le1 & - & - \\
\hline |l & $\overline{5}$ & $1.114 \mathrm{E} \overline{\mathrm{A}}$ & in & 1,150 & 3,0021 & $1.031 \mathrm{ti}$ & $3.7132 \%$ & $1.02 \mathrm{ES}$ & a 79411 & - & - & - & - \\
\hline 1ו & 4.에 & 1 . 년? & $.3624 \%$ & 1. & :i.i1.702 & 10312 & 3.56490 & 1.0200 & 3.7. & $1,0 \leqslant 3 \bar{i}$ & $3.75: 1.4 d$ & 1.1300 & $3 . \bar{i} 801 \mathrm{t}$ \\
\hline 3750 & 3. & $1.13 \mathrm{H}$ & 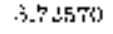 & 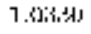 & i.T. & 1.0250 & 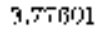 & 1.01250 & 0.19110 & & - & $\cdots$ & \\
\hline Uإن & . 1.00 & $1 . \mathrm{L}=1 \mathrm{~K}$ & $3.7 \div 541$ & $14 H$ & A.T THE & 1. $-125 \%$ & 7.90118 & $1.1124]$ & 3.52875 & $1,020 \mathrm{Q}$ & $0,2+418$ & 1.0023 & 0.54950 \\
\hline Hou & 3 & 1.102 & $\operatorname{siT54S}$ & 1. 1. & $3.54 \cdot 46.7$ & 1.[I" & 4.8005 & 1.1198 & $8 \times 47 T 1$ & & & & \\
\hline 1250 & 100 & $1.67 \mathrm{~B}$ & 3 set & 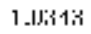 & ithirkifi & 1. & 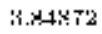 & 1.11230 & $3.57 \div, 19$ & 1.5218 & נ, 40122 & i & 7.50454 \\
\hline 4250 & (0) & 1,0709 & 30529 & 1.0213 & 3.54012 & 1.1 ENS & 3.8705 & $1.0 \div 21$ & 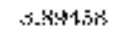 & 1. & i.silfits & - & - \\
\hline $\mathbf{s} 500$ & 4,600 & 1,004 & $3 \dot{s} \mathrm{rij}$ & 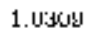 & 380121 & $1.1 \geqslant 54$ & 3.51206 & T 17 & 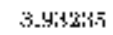 & 7.G2017 & i. $9540 \mathrm{H}$ & $1.020 \mathrm{~F}$ & 39.9942 \\
\hline 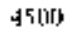 & 5 & 1,0511 & 3,69437 & $1.02 i z$ & 390151 & 1. & 3,02210 & 1.0211 & SUd72 & 7. & 3.0635 & 1.21211 & 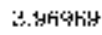 \\
\hline JTEL & 1.jull & 3. 0 sild & 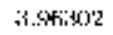 & $1.11 \div 4$ & (15BST4 & J 1.0251 & 3,07972 & 1. 10njos & 309002 & 1,5000 & 4,60754 & 1,002 & 4.00000 \\
\hline $4 \pi 5$ & 7.6)। & הדיםם נ & 8. & 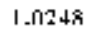 & 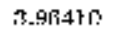 & $1, n \geq 10$, & 9,9740 & 1. .0204 & +401700 & i & 4,11714 & 1. (12)! & 4.0201 \\
\hline 5010 & נ. Ju & 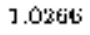 & 1.085 & 1 . $11 \leq 54$ & $1 . L 2365$ & 1.0k7 & J OALEK & تّاب! & $4-117+72$ & 1,0145 & 4,0H1 & 1.11795 & 4.01827 .7 \\
\hline 3040 & 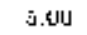 & 3. & 1.0815 & 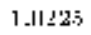 & A.J'LET & 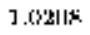 & 4 OKislu' & 1. Il1 & f.115, & 1.62010 & A.0RA:1 & itro' & 4. 1 mog \\
\hline $5 \geq 50$ & 4,00 & 1, 1,1900 & $4.0947 \mathrm{~J}$ & 1 _. $1 \geq 211$ & 1.086115 & 1.0140 & 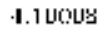 & 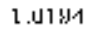 & 1.31121 & 1.094 & 4.11. & יומנוגום. & $1.176 \% 1$ \\
\hline 3260 & 5,00 & 1,013 & 4,LS1ذ1 & $1.0 \leq 02$ & A_LEsol & 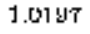 & 1.040132 & 1.148 & 1.J 1.30: & $7.09: 48$ & הברולנ 1.1 & ו & 1.12014 \\
\hline ลิ510 & 4.1711 & $1.0 \mathrm{LA} \overline{\mathrm{i}}$ & $4.1+179$ & (10101010 & 4.14541 & 1,1288 & A. $15: 40$ & $\bar{i}, 0102$ & 4.19504 & ב & $-1,100 \mathrm{~d}$ & $1.01 \% 1$ & 4.17154 \\
\hline 5 & ร.ภ117 & 1,000 & 4.15850 & 1,0179 & 4.1434 & 1,1198 & t. 15541 & 1.0106 & 4.16005 & 1.0906 & 1.17197 & $1 . \Delta 1 \%$ & 4.17396 \\
\hline & 1.U1J & 1.016 & +.1.564F & t.IIfHA & 4.210044 & 7.01 और 1 & 4.2105 & 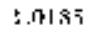 & 4.2167 & 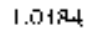 & 4.22714 & 1. IIIR2 & $4.22 \pi 57$ \\
\hline נינ:5is & 5.010 & 3.]-1.0 & L. Ly.]Jy & גונן & 1.14560 & 1 & ה & ד.thl ins & 1.21954 & $7.07 \times 5$ & 1.1212+2 & 194:17 & 1.2-5.14 \\
\hline 6000 & 1.010 & 1. & 1.214 & I. I I I I & 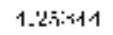 & 7.0158 & T. TH & 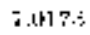 & 1.26yrs & 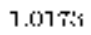 & 1.21.1. & ניהד רני. ד & 1.274H \\
\hline Bon & 500 & 1,0115 & 4,24731 & 1.0104 & 4.23107 & 1,0100 & 4.201040 & דיד & $4.270 \mathrm{de}$ & 1.017 & . 2516 & اנה 1.01. & 1.254 .78 \\
\hline ג'انيز1) & $4.00^{\circ}$ & 1,115 & t. 201\%1 & 1.10130 & $20031 \mathrm{~d}$ & 1.0135 & .3130 & 3.1416.5 & $1 . j 2 u T \pi$ & 1. & A & 1.2765 & 1.323 \\
\hline 30 & 5,000 & 1,0005 & 4.30820 & $1,6 \perp 12$ & 4,30184 & 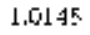 & 4.31313 & 5.01, لـ & \pm 92174 & $1, \square] \omega \perp$ & $1,32 \mathrm{~d} / \mathrm{k}$ & 1.0161 & 4.350 \\
\hline 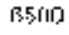 & 400 & 1,0150 & $4,34 \times 10$ & 1,0115 & 4.9327 & $1,010 \mathrm{i}$ & 4,50310 & $1,01 \div 4$ & 496897 & (1) & $-3 \pi 1 \geqslant 0$ & 1.0140 & 4.55176 \\
\hline 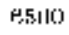 & E. IIIII & 1.8013 & 48479 & 1. 6942 & $4011 \%$ & 1,0125 & 4.511224 & $1.50 \%$ & דיוס & $1,018 \mathrm{i}$ & $4,52 \pi 2$ & 1.01 .35 & $4 \sin 11$ \\
\hline 67.50 & 1.UL:: & 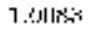 & 4.644F: & 1 604,2 & 4. .3F+41 & $7.6 \mathrm{~L}]$ & 441947 & 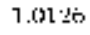 & 1.11501 & 1,0125 & $4.41 T i$ & 1.11734 & 4.41814 \\
\hline $6-50$ & E.lllt: & 7.AIILA & 4.8H:F1 & 1. Hhe & 4.19729 & 1.616:4 & $4.411: 14$ & צ'ום & $1.11 \mathrm{RH}$ & 7.0115 & 4.41515 & 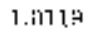 & +.41906 \\
\hline 7ี1110 & 1.Jlli: & (1) & A.167ו11 & i & +.AARLS & $7.010 \%$ & 4-4intin & $7.01 \mathrm{ks}$ & 1.15409 & & 4.4Fרי & 1.11700 & 4_tFERAt \\
\hline Fullo & 5.140 & "צרנונוג 1 & 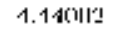 & 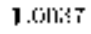 & - A-4ETS & 1.(1) & 4.45 & ה & 1. $\$ 24$ & i & 4,4 ;il & 1. & 4.4451 \\
\hline iscou & 4.00 & 1 _LUUA & 1.14650 & 105 & - 1. & 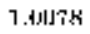 & 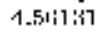 & $7.00 \mathrm{k}$ & 1.6020 & 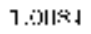 & 1.5IBE! & 1.11655 & f \\
\hline 750 & 500 & 0.5482 & 1. 185625 & 1.0 lin' 4 & 4.4C:14 & 1. (j)14:1 & 4 -5il:22; & 7. & +.F̈H $1 \mathrm{~B}$ & $1.005 \%$ & 4.5n: & I. IIIFA & 4. Brotin \\
\hline 700 & 4.0 & 1.0108: & -1.50101 & Lout 1 & 5ـ & 7.4U5y & 1.5.15:17 & 7 .ougsts & 1.51478 & 1-إلاقة. & $1.5=014$ & 1. L15s & 1.50088 \\
\hline 730 & 5.00 & 1]-14 & 1.520152 & 10 & A.ำล & 7. & 1.5457 & 5 רווة. & $4.7+4.57$ & 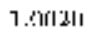 & 4.5.11\% & 1.114:101 & 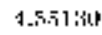 \\
\hline 7850 & $4.0 \underline{1}$ & (1.0970 & 小.58\11 & c.ogrs & 1.754h & 1. 1. & $450-47$ & 7.0lin & +. F:0i17 & 10\% & 4.61154:4 & 1. Linos & A. Fis 3 -1 \\
\hline 7.50 & ) & 0.4918 & 4.57.6. & 0,900 & 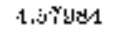 & 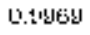 & A_Eeste & U.9.7s & 1.50100 & 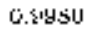 & A.EuSO & o.[1]) & 1.50310 \\
\hline Bnारण & +1.01 & 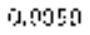 & 4,02403 & $0,50.37$ & - & LAtuks & A.HAR & 1.9005 & 1.6. $6 \% 1$ & Butbst & A.t.ts 37 & O.00St & 1.5.15s \\
\hline 年 & (5.0. & 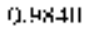 & 4. Filitit & $10.94,49$ & 4,132981 & C., $40 \geq 4$ & $4.02 \mathrm{J01}$ & 0.5005 & $4,0.3923$ & $\Gamma, 9040$ & 4,03459 & D.4041 & A. 0074 \\
\hline 8270 & $4,01$. & D_L954 & 4. HAAT: & L.: & $4 \cdot 3202$ & ogatio & 4. liand & $19,40 \%$ & $4.184 \times 1$ & $0,091 \mathrm{~s}$ & $4,68 \% 1 ! \mathrm{i}$ & D.1015 & $-1,04059$ \\
\hline 8250 & r.jo & 0. & 1.66526 & 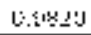 & teter & ㅇ․년독 1 & 1.fisuro & $0.95-3$ & L.61:246 & 0.9891 & $4,970,7$ & 0.7804 & 4. Firoty \\
\hline
\end{tabular}

is the large sensitivity of $R$-factors to the effective temperature $\left(R \sim T_{\text {eff }}^{3}\right)$ and their slight dependence on the secondary atmospheric parameters. Therefore, the uncertainties in the derived effective temperature associated to errors in the gravity and metallicity assignment are small compared to those due to the determination of the bolometric and monochromatic fluxes.

The monochromatic fluxes are obtained by applying the relation

$$
F\left(\lambda_{\mathrm{IR}}\right)=q\left(\lambda_{\mathrm{IR}}, T_{\mathrm{eff}},[\mathrm{Fe} / \mathrm{H}], g\right)\left[F_{\mathrm{cal}}\left(\lambda_{\mathrm{IR}}\right) 10^{-0.4\left(m-m_{\text {cal }}\right)}\right]
$$

where $m$ and $m_{\text {cal }}$ are, respectively, the magnitudes of the problem and standard star, $\lambda_{\mathrm{IR}}$ is the selected wavelength at the infrared (which should be close to the maximum transmission of the photometric band), $F_{\text {cal }}\left(\lambda_{\text {IR }}\right)$ is the absolute flux of the standard star at $\lambda_{\mathrm{IR}}$, and $q\left(\lambda_{\mathrm{IR}}, T_{\mathrm{eff}},[\mathrm{Fe} / \mathrm{H}], g\right)$ is a dimensionless factor which corrects the effect of the different curvature of the flux density distribution, across the filter window, between the stan- dard and the problem stars (see Sect. 3.2 for further details).

Table 5. The influence of the reddening and the uncertainty in the Calibration of the Absolute Flux in the IR (CAFIR) on the temperatures derived using IRFM. The errors induced by the uncertainties on the absolute flux calibration are slightly overestimated, since the change in the bolometric flux has not been taken into account

\begin{tabular}{|c|c|c|c|c|c|c|}
\hline & & \multicolumn{5}{|c|}{ Error on $T_{e f}(\%)$} \\
\hline Input parameter & Eттоr & 3500 & 4000 & 5000 & 6000 & 7500 \\
\hline $\bar{E}(\bar{B} \cdot V)$ & $0.05 \mathrm{mag}$ & 2.1 & 1.6 & 2.6 & 3.6 & $\overline{4.5}$ \\
\hline CAFlR J $[\mathrm{Fe} / \mathrm{H}]=0.0$ & $3 \%$ & 2.75 & 3 & 2 & 1.4 & 1.2 \\
\hline CAFIR J $[\mathrm{Fe} / \mathrm{H}]=-\mathrm{M} \mathrm{O}$ & $3 \%$ & 3.25 & 2.75 & 2 & 1.4 & 1.2 \\
\hline CAFIR H $[\mathrm{Fe} / \mathrm{H}]=0 . \mathrm{A}$ & $4 \%$ & 6 & 1.6 & 1.4 & 1.2 & 1.2 \\
\hline CAF[R $H[F \in / H]=-3.0$ & $4 \%$ & 1.75 & 2 & 2.75 & 1.2 & I. 2 \\
\hline CAFLR K $[$ Fe/H $]=0.0$ & $4 \not$ & 2.4 & 1.4 & 1.4 & 1.2 & 1.2 \\
\hline CAFIR $K[\mathrm{Fe} / \mathrm{H}]=-3.0$ & $4 \%$ & 1.6 & 1.6 & 1.4 & 1.2 & 1.2 \\
\hline CAFIR mean & - & 2.0 & $\overline{1.7}$ & 1.6 & 1.25 & 1.2 \\
\hline
\end{tabular}

The separation of observational inputs and theoretical factors for the implementation of the IRFM is provided 


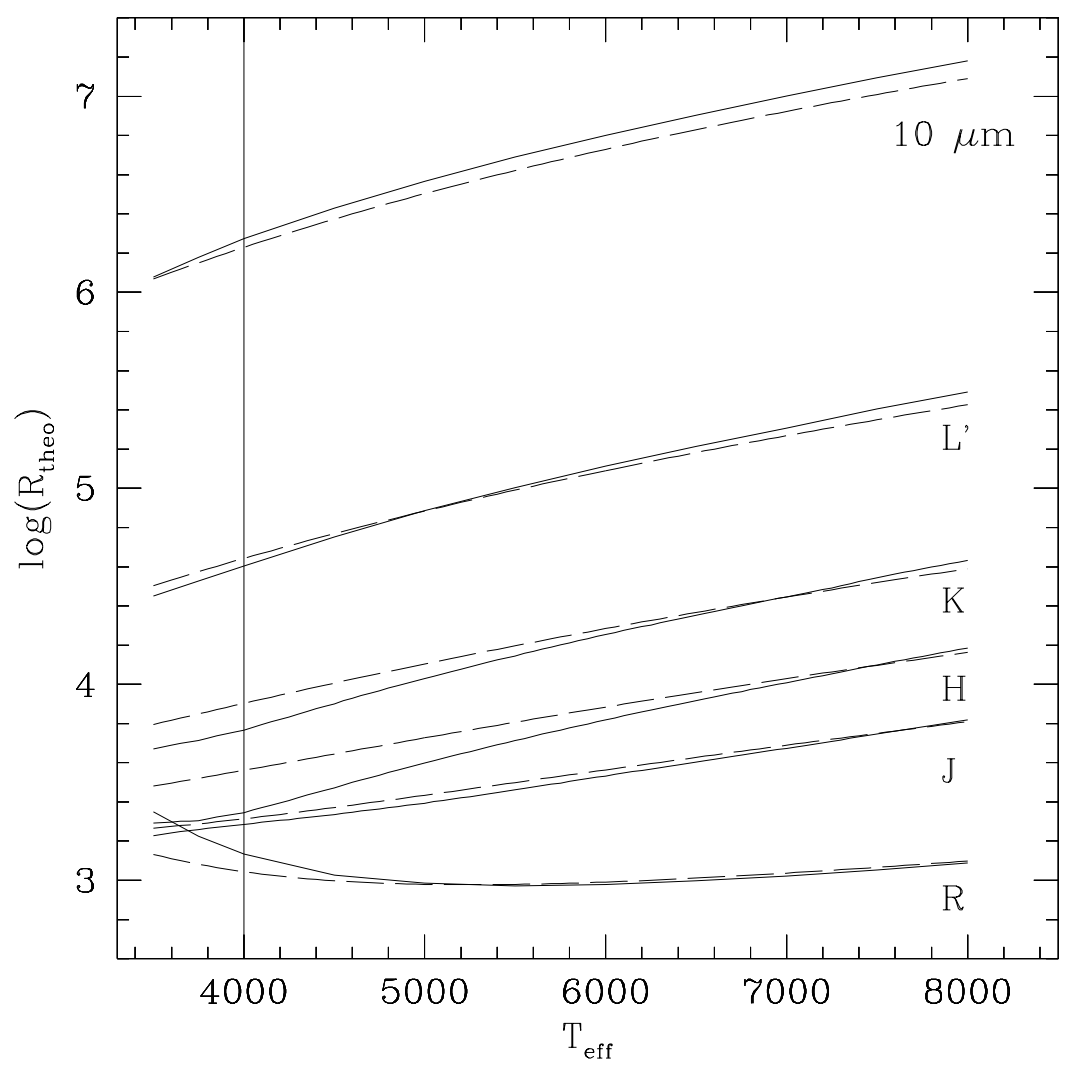

Fig. 1. $R_{\text {theo }}$ factors computed using solar metallicity models developed by Kurucz (1991). The wavelengths considered are $\lambda_{R}=790 \mathrm{~nm}, \lambda_{J}=1272.5 \mathrm{~nm}, \lambda_{H}=1635 \mathrm{~nm}, \lambda_{K}=2175 \mathrm{~nm}, \lambda_{L^{\prime}}=3690 \mathrm{~nm}$, and a point in the far IR at $10 \mu m$. The dashed lines correspond to $R_{\text {theo }}$ factors derived from blackbody flux densities. The vertical line $\left(T_{\text {eff }}=4000 \mathrm{~K}\right)$ shows the lower limit of applicability of IRFM with these models

Table 6. Mean accidental errors of the effective temperature derived applying the IRFM in the band $K$

\begin{tabular}{|c|c|c|c|c|c|c|}
\hline & & \multicolumn{5}{|c|}{ Error on $T_{e /}(\%)$} \\
\hline Iuput parameter & Error & $3500 \mathrm{~K}$ & $4000 \mathrm{~K}$ & $5000 \mathrm{~K}$ & $6000 \mathrm{~K}$ & $7500 \mathrm{~K}$ \\
\hline$q R_{t h e \psi}$ & $5 \%$ & 3 & 1.5 & 1.5 & 1.5 & 1.5 \\
\hline $\log (g)$ & $0.5 \mathrm{dex}$ & 2 & 1.5 & 0.1 & 0.1 & 0.1 \\
\hline$[F e / H]$ & 0.3 dex & 1 & 0.75 & 0.15 & 0.15 & 0.15 \\
\hline Total & & 3.3 & $\overline{2}$ & 1.5 & 1.5 & 1.5 \\
\hline
\end{tabular}

by substituting relation (3) in Eq. (2) as follows,

$$
\begin{gathered}
\frac{F_{\mathrm{Bol}}}{F_{\text {cal }}\left(\lambda_{\mathrm{IR}}\right) 10^{-0.4\left(m-m_{\text {cal }}\right)}}= \\
=q\left(\lambda_{\mathrm{IR}}, T_{\mathrm{eff}},[\mathrm{Fe} / \mathrm{H}], g\right) R_{\mathrm{theo}}\left(\lambda_{\mathrm{IR}}, T_{\mathrm{eff}},[\mathrm{Fe} / \mathrm{H}], g\right)
\end{gathered}
$$

Once $[\mathrm{Fe} / \mathrm{H}]$ and $\log (g)$ are known for a certain star, the observational quantities on the left-hand side of Eq. (4) determine the star's effective temperature by comparing to the theoretical information from models on the right-hand side. It is worthy to notice that in a strict sense, models are also needed to obtain the integrated flux in order to
Table 7. Comparison between the temperatures derived in the present work (Col. 2) and those derived by Saxner \& Hammarbäck (1985) (Col. 3). The mean difference $T_{\mathrm{IRFM}}-T_{\mathrm{SH} 85}$ is $5 \pm 63 \mathrm{~K}$

\begin{tabular}{cccrr}
\hline Star & $T_{\text {IAFM }}(\mathrm{K})$ & $T_{\text {SHS }}(\mathrm{K})$ & $\Delta T$ & $\Delta T \cdot(\mathrm{K})(\%)$ \\
\hline HR0458 & 6155 & 6140 & 15 & 0.24 \\
HR0483 & 5874 & 5810 & 64 & 1.09 \\
HR0937 & 5996 & 5890 & 106 & 1.77 \\
HR1101 & 5998 & 5940 & 58 & 0.97 \\
HR1543 & 6482 & 6470 & 12 & 0.19 \\
HR1729 & 5847 & 5810 & 37 & 0.63 \\
HR1983 & 6260 & 6260 & 0 & 0.00 \\
HR2085 & 7013 & 7160 & -147 & -2.10 \\
HR2852 & 7020 & 7050 & -40 & -0.57 \\
HR2943 & 6579 & 6640 & -61 & -0.93 \\
HR4540 & 6095 & 6120 & 25 & -0.41 \\
HR4785 & 5867 & 5810 & 57 & 0.97 \\
HR5447 & 6770 & 6760 & 10 & 0.15 \\
HR5634 & 5571 & 6570 & 1 & 0.02 \\
HR5868 & 5897 & 5890 & 7 & 0.12 \\
HR5914 & 5774 & 5870 & -96 & -1.66 \\
HR5933 & 6233 & 6220 & 13 & 0.21 \\
HR8905 & 5954 & 5870 & 34 & 1.41 \\
\hline
\end{tabular}




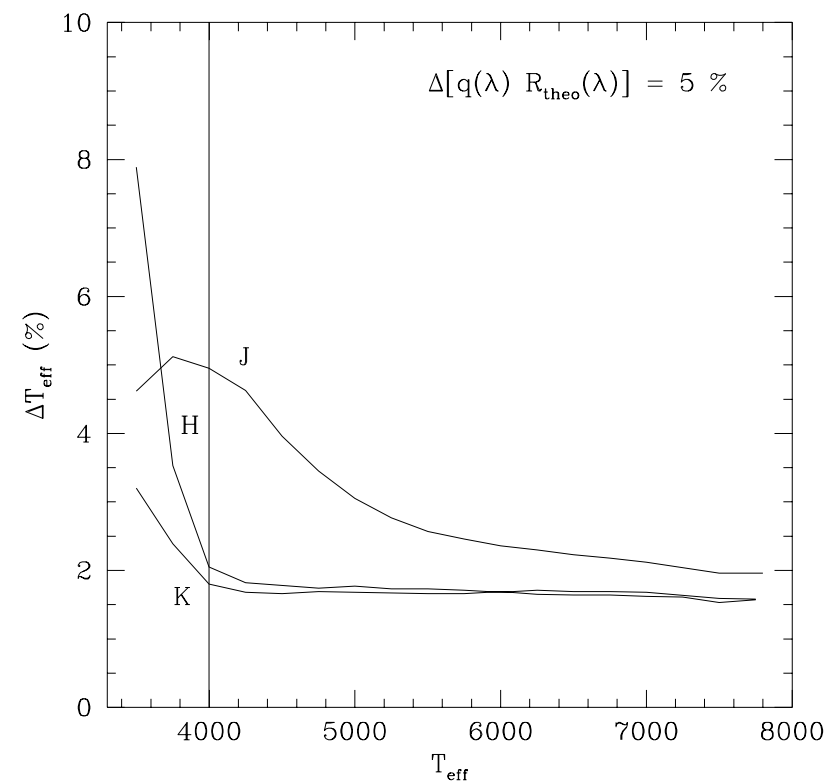

Fig. 2. Uncertainty on the IRFM temperature induced by an error of $5 \%$ in the observational quotient $\frac{F_{\text {Bol }}}{F_{\text {cal }}\left(\lambda_{\text {IR }}\right) 10^{-0.4\left(m-m_{\text {cal }}\right)}}$

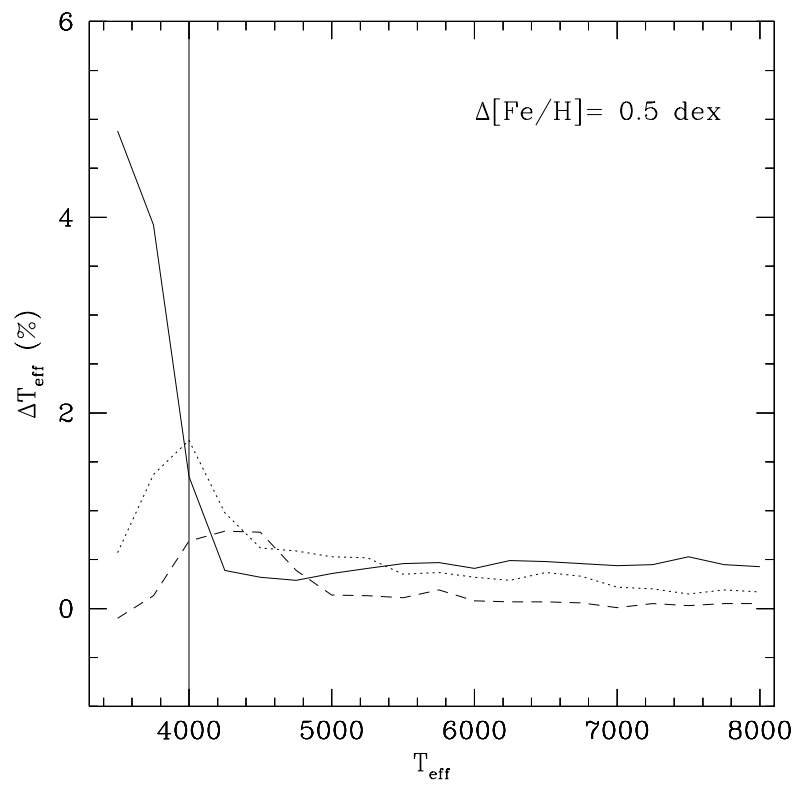

Fig. 3. Uncertainty on the IRFM temperature induced by an error of 0.5 dex in $[\mathrm{Fe} / \mathrm{H}]$. Solid line: change from $[\mathrm{Fe} / \mathrm{H}]=0$ to $[\mathrm{Fe} / \mathrm{H}]=-0.5$; dotted line: change from $[\mathrm{Fe} / \mathrm{H}]=-1$ to $[\mathrm{Fe} / \mathrm{H}]=-1.5$; dashed line: change from $[\mathrm{Fe} / \mathrm{H}]=-2.5$ to $[\mathrm{Fe} / \mathrm{H}]=-3$ complete the missed flux in the UV and far IR, however, these corrections are small (see for instance Petford et al. (1991) and Paper II).

\section{Model information: The $R-$ and $q$-factors}

The theoretical flux density distributions used in this work to implement the IRFM were obtained using $\mathrm{Ku}$ rucz's $(1991,1993)$ new models. Blackwell and LynasGray (1994) have also used them for the application of the IRFM. These models are widely described in Kurucz (1991). Here we briefly summarize their features concerning the aims of the present programme:

1. The major difference concerning opacities is the use of new iron group atomic lines, which are expected to diminish the problem of the missing opacity in the UV (Magain 1987). The computed opacities consider the effect of 58 million lines both atomic and molecular (which increases in a factor of roughly 30 the number in old models). Diatomic molecules considered include $\mathrm{H}_{2}, \mathrm{SiO}, \mathrm{CH}, \mathrm{NH}, \mathrm{OH}, \mathrm{MgH}, \mathrm{SiH}, \mathrm{C}_{2}, \mathrm{TiO}, \mathrm{CN}$ and CO. Regrettably the effects of triatomic molecules, as water vapor, are not considered. In the range 1.75-2.1 $\mu m$ the contribution to the opacity from water vapour has great influence on the IR flux of cool stars $\left(T_{\text {eff }} \leq\right.$ $4500 \mathrm{~K}$ ), specially for solar abundance. This fact sets a lower limit at late $\mathrm{K}$ stars for the applicability of the IRFM using these models (see Sect. 5).

2. The physics remains essentially the same that in older Kurucz's models (see Kurucz 1979a, b) since the problem of the opacity has been paid major attention. However, the new models contain slight improvements in the treatment of convention including approximative overshooting. The mixing length to scale height ratio $(\alpha / H)$ adopted is 1.25 . The solar metal abundances are those derived by Anders \& Grevesse (1989). The microturbulent velocity is $2 \mathrm{~km} / \mathrm{s}$.

3. From a practical point of view, the new models sample physical parameters in a denser grid: Abundances are sampled in 0.1 dex steps from $[\mathrm{Fe} / \mathrm{H}]=1.00$ to $[\mathrm{Fe} / \mathrm{H}]=-0.5$, and in 0.5 dex steps from $[\mathrm{Fe} / \mathrm{H}]=-0.5$ to $[\mathrm{Fe} / \mathrm{H}]=-4.0$. Gravities are sampled in 0.5 dex steps and temperatures in $250 \mathrm{~K}$ steps under $8000 \mathrm{~K}$.

4. Model fluxes are sampled in 1221 points covering a wavelength interval from 9 to $160000 \mathrm{~nm}$. The resolution ranges within $1 \mathrm{~nm}$ in the UV, $2 \mathrm{~nm}$ in the visible and the band $J, 5 \mathrm{~nm}$ in the band $H$ and $10 \mathrm{~nm}$ in the band $K$. The sampling in the near IR implies a remarkable improvement as far as previous models are concerned, which allows to avoid interpolations.

\section{1. $R_{\text {theo }}\left(\lambda_{\mathrm{IR}}\right)$ factors}

The flux density distributions of models described in the previous section have been used to calculate $R_{\text {theo }}\left(\lambda_{\mathrm{IR}}\right)$ 
Table 8. Comparison between the temperatures derived in the present work (Col. 2) and those derived by Magain (1987) (Col. 3 ). The mean difference $T_{\mathrm{IRFM}}-T_{\mathrm{M} 87}$ is $112 \pm 56 \mathrm{~K}$

\begin{tabular}{lccrr}
\hline \multicolumn{1}{c}{ Star } & $T_{\text {IRFM }}(\mathrm{K})$ & $T_{\text {MS7 }}(\mathrm{K})$ & $\Delta T(\mathrm{~K})$ & $\Delta T(\%)$ \\
\hline HD19445 & 6030 & 5933 & 117 & 1.93 \\
HD64000 & 5441 & 5370 & 71 & 1.36 \\
HD74000 & 6224 & 6135 & 89 & 1.40 \\
HD84937(2) & 6330 & 6210 & 120 & 1.90 \\
HD94028(3) & 6001 & 5777 & 224 & 3.73 \\
HD108177 & 6034 & 6026 & 8 & 0.01 \\
HD132475 & 5788 & 5614 & 174 & 3.00 \\
HD140283 & 5691 & 5607 & 84 & 1.47 \\
BD +42 2667 & 6059 & 5955 & 104 & 1.71 \\
HD201891 & 5909 & 5761 & 148 & 2.56 \\
HD219617 & 6012 & 5918 & 94 & 1.56 \\
\hline \hline
\end{tabular}
(1) G090-025
(2) G043-003
(3) G058-025
(4) GE80-024

Table 9. Comparison between the temperatures derived in the present work (Col. 2) and those derived by Bell \& Gustaffson (1989) (Col. 3). The mean difference $T_{\mathrm{IRFM}}-T_{\mathrm{BG} 89}$ over $4500 \mathrm{~K}$ is $-49 \pm 75 \mathrm{~K}$

\begin{tabular}{cccrr}
\hline Star & $\bar{T}_{I A F M}(\mathrm{~K})$ & $T_{B L 44}(\mathrm{~K})$ & $\Delta T(\mathrm{~K})$ & $\Delta T(\%)$ \\
\hline HR219 & 5817 & 5839 & -22 & -0.38 \\
HR753 & 4718 & 4775 & -57 & -1.21 \\
HR4785 & 5867 & 5867 & 5 & 0.10 \\
HR4983 & 5964 & 6024 & -60 & -1.01 \\
HR7503 & 5763 & 5826 & -63 & -1.09 \\
HR7504 & 5767 & 5664 & 103 & 1.79 \\
HR4496 & 5342 & 5552 & -210 & -3.93 \\
HR7462 & 5227 & 5253 & -26 & -0.50 \\
HR1325 & 5079 & 5114 & -35 & -0.69 \\
HR1084 & 5076 & 5156 & -80 & -1.58 \\
HR8832 & 4805 & 4896 & -91 & -1.89 \\
HR3085 & 4323 & 4463 & -140 & -3.24 \\
HR8086 & 3865 & 4252 & -387 & -10.01 \\
\hline \hline
\end{tabular}

factors, as defined in Eq. (2). The closest wavelengths to the effective wavelengths of the $J, H$, and $K$ photometric bands sampled by the models were selected $\left(\lambda_{J}=1272.5\right.$ $\mathrm{nm}, \lambda_{H}=1635.0 \mathrm{~nm}$ and $\left.\lambda_{K}=2175.0 \mathrm{~nm}\right)$. The effective wavelengths were computed considering the instrumental response of the TCS system (Paper I), and the atmospheric transparency (Manduca \& Bell 1979; Glass 1985; Mountain 1983). Tables 1-3 contain the theoretical values of $\log (R)$ for these three wavelengths. Effective temperatures cover the range $3500-8250 \mathrm{~K}$, surface gravities cover the range $\log (g)=(3.5,5.0)$, and metallicities cover the range $(0.5,-3.5)$.

Figure 1 shows the $\log (R)$ factors for solar metallicity. In addition to $R$-factors corresponding to the three wavelengths selected in this work, those corresponding to the $R$ band (Johnson 1966) at $\lambda_{R}=790 \mathrm{~nm}, L^{\prime}$ from TCS system (Paper II) at $\lambda_{L^{\prime}}=3690.0 \mathrm{~nm}$, and a far

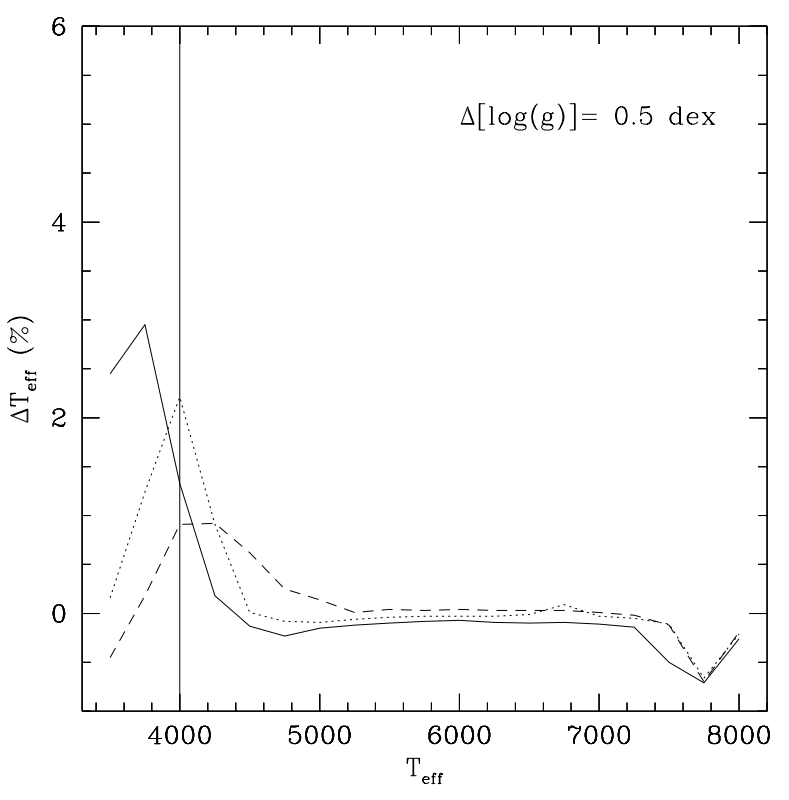

Fig. 4. Uncertainty on the IRFM temperature induced by an error of 0.5 dex in $\log (g)$. Solid line: $[\mathrm{Fe} / \mathrm{H}]=0.0$; dotted line: $[\mathrm{Fe} / \mathrm{H}]=-1$; dashed line: $[\mathrm{Fe} / \mathrm{H}]=-2$

IR point at $10004.0 \mathrm{~nm}$ have been also plotted to display their overall properties. The correlation of gradient $\Delta \log \left(R_{\text {theo }}\right) / \Delta T_{\text {eff }}$ with wavelength may be appreciated in the figure (i.e. the sensitivity of $R$-factors to temperature increases with wavelength). The relation $\log \left(R_{\text {theo }}\right)-$ $T_{\text {eff }}$ is double-valued for the band $R$, which implies that it is useless to apply the IRFM in the considered $T_{\text {eff }}$ range.

Among the three wavenlengths considered in this work, the $R_{J}$ factors are the least sensitive to temperature, especially for $T_{\text {eff }}$ lower than $5000 \mathrm{~K}$. The sensitivities of the $R_{H}$ and $R_{K}$ are comparable, although as will be discussed in Sect. 5, temperatures lower than $4000 \mathrm{~K}$ derived using $R_{H}$ are less reliable, due to the uncertainty associated to the minimum of the $\mathrm{H}^{-}$opacity reached in this band. The variations induced by the change in metallicity or surface gravity are only important for $T_{\text {eff }}$ lower than $4250 \mathrm{~K}$. In particular, the variation of $R$-factors in the range $\log (g)=4-5$ is almost negligible. For this reason, the assignation of gravity has been done in a somewhat rough fashion, which satisfies nevertheless the accuracy requeriments of this work.

\section{2. $q\left(\lambda_{\mathrm{IR}}\right)$ factors}

The use of broad band photometry to obtain the IR monochromatic fluxes requires the application of the 
so-called $q$-factors introduced in Eq. (3), and defined as

$$
q\left(\lambda_{\mathrm{IR}}\right)=\frac{\int_{\lambda_{1}}^{\lambda_{2}} K_{\mathrm{cal}}\left(\lambda, \lambda_{\mathrm{IR}}\right) T(\lambda) \mathrm{d} \lambda}{\int_{\lambda_{1}}^{\lambda_{2}} K\left(\lambda, \lambda_{\mathrm{IR}}\right) T(\lambda) \mathrm{d} \lambda},
$$

where $T(\lambda)$ is the instrumental transmission of the photometric system, which includes the detector response, the optical system of the telescope and photometer, and the absorption of the atmosphere. The function $K\left(\lambda, \lambda_{\mathrm{IR}}\right)$ is the stellar flux density normalized to the value of flux density at $\lambda_{\mathrm{IR}}$, and $\left(\lambda_{1}, \lambda_{2}\right)$ is the bandpass of the system (see Appendix 1 in Paper II for further details). The $q$-factors allow to cope with the problem of deriving the flux in a specific wavelength from the filter-integrated flux in the whole photometric band. An optional approach to this problem is described in Saxner \& Hammarbäck (1985).

Ideally, $q$-factors should be determined from spectroscopic data, considering a set of stars sampling homogeneously the domain of physical parameters in $T_{\text {eff }}, \log (g)$ and $[\mathrm{Fe} / \mathrm{H}]$. Unfortunately the data-base of registered IR spectra is insufficient to make this approach realistic. In practice, we can rely on a grid of models to compute $q$ factors. Tables 1-3 contain $q$-factors for bands $J, H$ and $K$, ordered according to temperature, gravity and metallicity. The physical parameters adopted to generate the model for the calibration star (Vega) were $T_{\text {eff }}=9610 \mathrm{~K}$, $\log (g)=3.95,[\mathrm{Fe} / \mathrm{H}]=-0.25$. Note that the $q$-factors imply secondary corrections in most of the range studied.

\subsection{Sensitivity of $q \times R$ to the effective temperature}

The separation of terms in Eq. (4) (i.e. theoretical elements in the right-handside, and observational data in the left-handside) provides a simple way of analysing the influence of errors on the derived temperatures. Tables 1-3 contain the calibration of $R$ - and $q$-factors generated with Kurucz's new models as a function of temperature, metallicity and surface gravity. These relations allow to determine the errors induced by the different variables on the derived temperatures.

Figure 2 shows the error in temperature induced by a variation of $5 \%$ in factor $q \times R$ (the theoretical counterpart to the quotient $\left.F_{\mathrm{Bol}} / F\left(\lambda_{\mathrm{IR}}\right)\right)$. As it may be appreciated, the change in temperatures derived using $R_{H}$ and $R_{K}$ factors is approximately constant over $4000 \mathrm{~K}: 1.5-2 \%$ for $T_{H}$ and $1.5 \%$ for $T_{K}$. The change of $T_{J}$ varies from $5 \%$ at $4000 \mathrm{~K}$ to $2 \%$ at $8000 \mathrm{~K}$. Hence, $R_{J}$ is the worse indicator of $T_{\text {eff }}$ for the application of the IRFM, and will only be used over $5000 \mathrm{~K}$.

Figure 3 shows the influence of an error of 0.5 dex in metallicity on the temperature derived applying the IRFM in the band $K$. Over $4200 \mathrm{~K}$ the average error is under $0.25 \%$.
Figure 4 shows the influence of an error of 0.5 dex in $\log (g)$ on the temperature derived in the band $K$. The changes in temperature are practically negligible between $4200 \mathrm{~K}$ and $7500 \mathrm{~K}$. Between $4000 \mathrm{~K}$ and $4200 \mathrm{~K}$ the change amounts $1-2 \%$. Over $7500 \mathrm{~K}$ the change may amount $-0.5 \%$.

\section{Observational inputs for the IRFM}

In the following paragraphs the different observational inputs which enter the application of the IRFM will be commented. First of all, we provide a description of the sample of stars collected for this program.

\subsection{The selection of the sample}

The stars of the sample were extracted mainly from three independent photometric surveys: Sandage \& Kowal (1986), Carney \& Latham (1987) and Schuster \& Nissen (1988). These works are involved with the study of different properties of halo field stars, and provide broad band $U B V$ and Strömgren photometry. The selected sample of stars covers the entire range of effective temperature and metallicity observed in the main sequence of globular clusters, and intermediate and old disk clusters (i.e. late spectral types of luminosity class $\mathrm{V}$ and VI). The stars of the sample range practically $0.3<(B-V)<1.7$, $0.1<(b-y)<1.0$, and $0.0<\delta_{0.6}(B-V)<0.3$, which roughly implies $-3.5<[\mathrm{Fe} / \mathrm{H}]<0.5$. After the measurement of IR photometry (Paper I), the stars with ( $V-$ $K) \geq 4.2$ were discarded since the practical limit in temperatures of the models is around this value (equivalent to $\sim 3500 \mathrm{~K}$ ). A subsample containing dwarfs, and all the subdwarfs from the Catalogue of Spectroscopic Abundances (Cayrel de Strobel et al. 1992) with $[\mathrm{Fe} / \mathrm{H}]<0$, observable from the Teide Observatory $\left(66^{\circ}>\delta>-28^{\circ}\right)$ has also been included in order to revise the published relations $[\mathrm{Fe} / \mathrm{H}]=f\left((b-y), m_{1}, c_{1}, \delta_{0.6}(U-B),(B-V) \ldots\right)$. The revisions will provide good estimates of the metallicity for the remainder of the stars of the programme (see Sect. 4.5). A number of stars in the sample are in the input catalogue of the Hipparcos mission. The sample also contains a group of metal poor stars whose parallaxes have been accurately determined (Sandage 1983; Laird et al. 1987; Van Altena et al. 1988).

The dwarfs and subdwarfs from Carney (1983a, b) not common to Paper I were incorporated in order to increase the sampling of the metallicity range for types F0-K0. It is noteworthy the good quality of Carney's photometry, and also the close similarity of TCS and CIT infrared systems. The dwarfs contained in the list of calibration stars of the TCS (Kidger 1992) not measured for this programme have also been incorporated into the sample. Some stars contained in the study of M dwarfs by Legget $(1992)$ with $(V-K) \leq 4.0$ were initially 
Table 10. Comparison between the temperatures derived in the present work (Col. 2) and those derived by Blackwell \& Lynas-Gray (1994) (Col. 3). The mean difference $T_{\mathrm{IRFM}}-T_{\mathrm{BG} 89}$ is $-31 \pm 58 \mathrm{~K}$

\begin{tabular}{lccrr}
\hline \multicolumn{1}{c}{ Star } & $T_{I R F M}(\mathrm{~K})$ & $T_{B L 94}(\mathrm{~K})$ & $\Delta T(\%)$ & $\Delta T(\mathrm{~K})$ \\
\hline HR0509 & 5388 & 5316 & -1.34 & 72 \\
HR0219 * & 5817 & 6044 & 3.90 & -227 \\
HR0937 & 5996 & 6042 & 0.77 & -46 \\
HR1101 & 5998 & 5977 & -0.35 & 21 \\
HR1325 & 5079 & 5163 & 1.65 & -84 \\
HR1729 & 5847 & 5947 & 1.71 & -100 \\
HR5447 & 6770 & 6763 & -0.10 & 7 \\
HR5634 & 6571 & 6617 & 0.70 & -46 \\
HR5986 & 6158 & 6169 & 0.18 & -11 \\
HR8905 & 5954 & 6050 & 1.61 & -96 \\
\hline
\end{tabular}

* This spectroscopic binary star has been discarded in the computation of the mean difference.

included in the sample, but their cool temperatures (3500-

$4000 \mathrm{~K})$ are well under limit of validity of the models due to the problems associated to the computation of molecular opacities (Kurucz 1991). Finally, some bright population I stars from Saxner \& Hammarbäck (1985) and Bell \& Gustafsson (1989), and population II stars from Arribas \& Martínez-Roger (1989), who provide temperatures obtained by applying the IRFM, have been included in the sample in order to make comparisons.

In summary, the final sample consists of nearly 500 stars. This number clearly surpasses that of the previous works devoted to the study of the scale of temperatures of low main sequence, especially those which analyse metallicity effects.

\subsection{The IR monochromatic fluxes}

The determination of monocromatic fluxes at a wavelength of the IR continuum requires, from the observational side, the measurement of IR photometry for the problem stars with respect to a standard whose absolute flux be well determined. By using the results summarised in Sects. 4.2.1 and 4.2.2, we have determined the monochromatic fluxes in $\lambda_{J}, \lambda_{H}$ and $\lambda_{K}$ for each star in the present sample, which are listed in Table 4.

\subsubsection{The IR photometry}

The programme of broad band photometry in the near IR is described in Paper I. There, the photometric system of the TCS was characterized and the transformation equations into/from different photometric systems were established. The $J, H$ and $K$ magnitudes were measured for $75 \%$ of the stars in the sample, with an accuracy in the order of $0.02 \mathrm{mag}$. For the remainder of the stars, the photometry was obtained from the literature (Carney 1983a, b; Legget 1992; Kidger 1992; Saxner \& Hammarbäck 1985; Bell \& Gustaffson 1989), after checking that these works had a similar level of accuracy.
The isolated effect of the photometric errors on the $T_{\text {eff }}$ determination can be inferred from Sect. 3.3, taking into account Eq. (4).

\subsubsection{The absolute calibration of the IR flux}

In Paper II, a semiempirical method was devised to determine the absolute calibration of the flux of Vega in the near IR (from $J$ to $L^{\prime}$ ). This absolute calibration sets on the same scale the temperatures derived applying the IRFM with new Kurucz's models and the mean direct temperatures derived from angular diameters measurements. When compared to the results of the only sofar empirical calibration, summarised by Mountain et al. (1985, M85), a clear trend with wavelength may be appreciated $(+0.2 \%$ in the band $J,-1.5 \%$ in the band $H$ and -4.5 in the band $K)$. This point suggests that the IR fluxes for Vega by M85 are overestimated towards the longest wavelengths. However, it is worth noticing the good agreement (within 1\%) with the semiempirical calibration for Vega provided by Walker \& Cohen (1992), the theoretical one by Dreiling \& Bell (1980) and the 'selfconsistent' calibration by Blackwell et al. (1991).

The errors in the absolute IR flux calibration have different effects on the temperatures derived by mean of the IRFM, depending on the photometric band (Table 5). The errors of the absolute IR flux calibration were estimated $3 \%$ in the band $J$, and $4 \%$ in the bands $H$ and $K$. Over $4000 \mathrm{~K}$ the effect of these errors is practically a shift in the zero point of the temperatures scale. Considering that the above errors correlate in the three bands, the shift in the zero point of the temperature scale would amount $1.2-1.7 \%$ over $4000 \mathrm{~K}$. However if, as it is most likely, the errors in the three bands are uncorrelated, the shift of the zero point is around $0.4-0.9 \%$. Although the indetermination of the zero point of the scale is common to all kind of methods used to derive temperatures, the method adopted in Paper II to fix the absolute calibration of the 


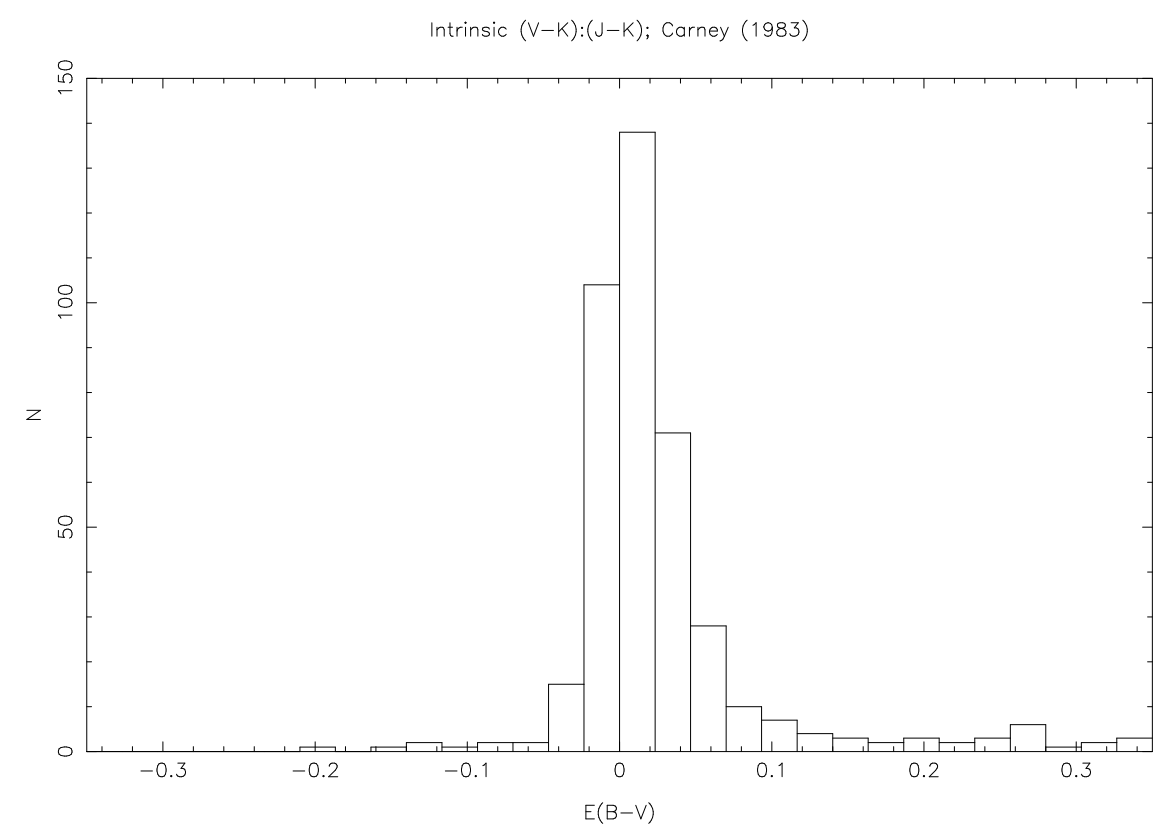

Intrinsic (b-y); Schuster and Nissen (1989)

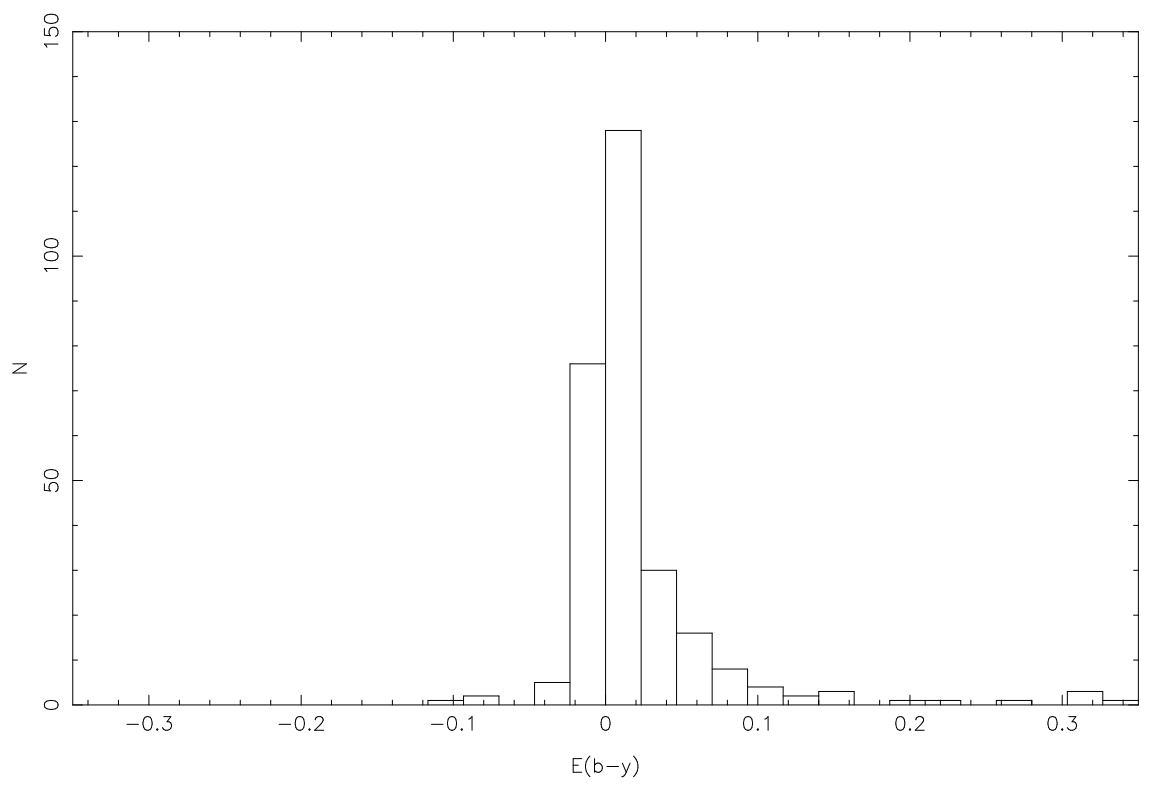

Fig. 5. Top: extinction histogram $(E(B-V))$ obtained applying to the stars of the sample the method based on the intrinsic colours $(V-K):(J-K)$ (Carney 1983). Bottom: extinction histogram $(E(b-y))$ obtained applying to the stars of the sample the method based on the intrinsic relations Strömgren photometry- $\beta$ index (Schuster \& Nissen 1989)

flux in the near IR was designed in order to minimise this error.

\subsection{The bolometric fluxes}

For the spectral types studied in the present work, nearly all the flux arriving at the edge of the earth atmosphere passes through the atmospheric windows. Petford et al. (1988) report an accuracy of the order of $2 \%$ when compar- ing $F_{\text {bol }}$ derived directly from calibrated spectra to $F_{\text {Bol }}$ obtained integrating $U B V R I$ photometry. Therefore, the bolometric flux might be obtained from broad band photometry for each star in the sample. However, photometric calibrations of the type provided by Blackwell \& Petford (1991) represent a more practical, and as accurate approach. Unfortunately, this calibrations do not include the effect of the metallicity, being only valid for Population I stars. In order to overcome this dificulty, we provided in 

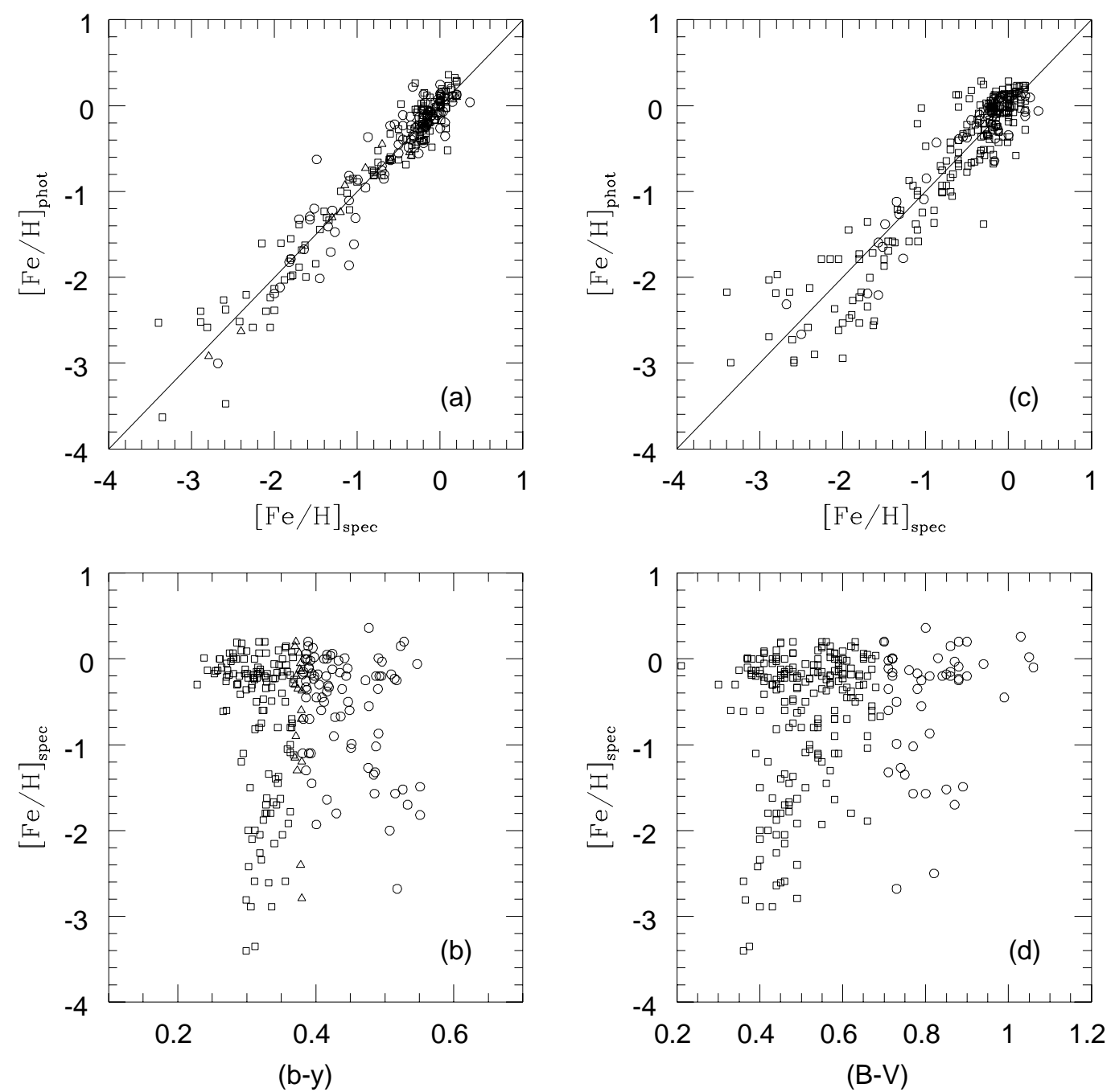

Fig. 6. a) Comparison between spectroscopic values of the metal abundance (Cayrel de Strobel et al. (1992) and Beers et al. (1991)) and values derived using the calibration by Schuster \& Nissen (1989) revised according to expressions (6) and (7). Circles: calibration for F stars, squares: calibration for G stars, triangles: stars in the overlapping range of F and G calibrations. b) Range of colour covered by the revised calibration by Schuster \& Nissen (1989). c) Comparison between spectroscopic values of the metal abundance (Cayrel de Strobel et al. 1992 and Beers et al. 1990) and values derived using the calibration by Carney (1979) revised according to expressions (9) and (10). Squares: $(B-V)>0.7$, circles: $(B-V)<0.7$. d) Range of colour covered by the revised calibration by Carney (1979)

Paper III calibrations of the bolometric flux as a function of $K,(V-K)$ and $[\mathrm{Fe} / \mathrm{H}]$ for main sequence stars of spectral types F, G and $K$, which grant the overall level of accuracy expected for the final temperatures derived in this work. It should be noted that these calibrations are ultimately based on the optical absolute flux calibrations of Vega by Hayes \& Latham (1975) and Tüg et al. (1977), and the IR absolute flux of Paper II. The bolometric fluxes assigned to the stars in the sample are listed in Table 4.

\subsection{The reddening correction}

Most of the stars in the sample should not be significantly affected by the interstellar absorption, since are distributed close in the solar neighbourhood. However, in some cases reddening corrections need to be applied. Therefore, $E(B-V)$ has been estimated for each star in the sample. When these values have been considered significant, the colours have been corrected according to the extinction law $\left(A_{\lambda}=f\left(A_{V}, \lambda\right)\right)$ compiled by Landolt-Börnstein (1982c). Two independent methods 

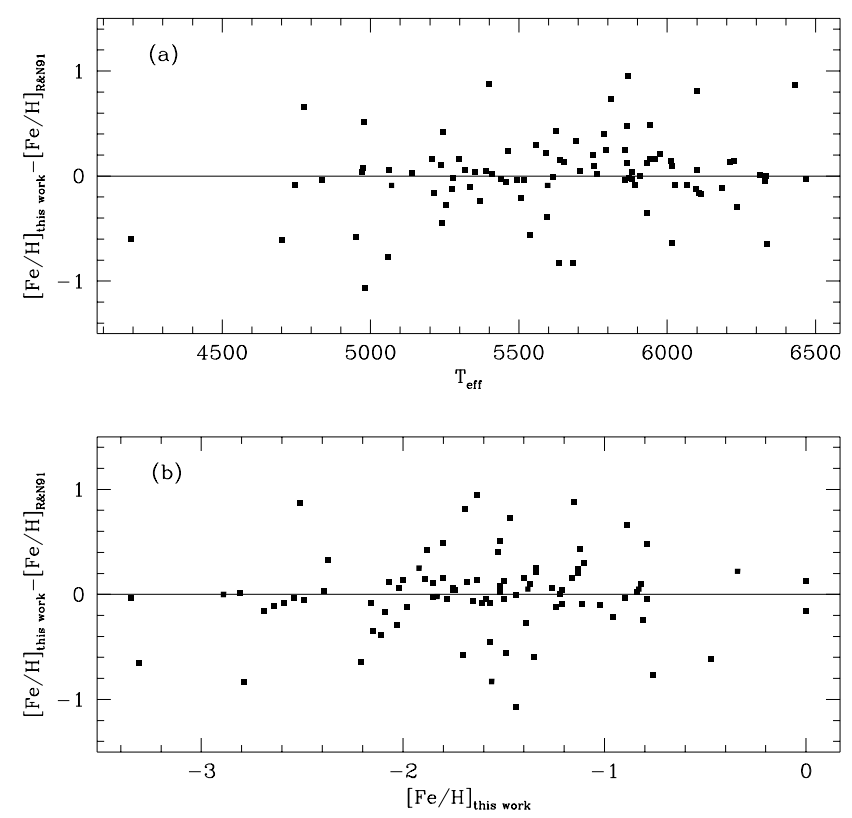

Fig. 7. a) Differences between the metallicities adopted in this work and those derived by Ryan \& Norris (1991) versus effective temperature. b) The same that in a) versus metallicity. The differences show no apparent trends either with temperature or metallicity
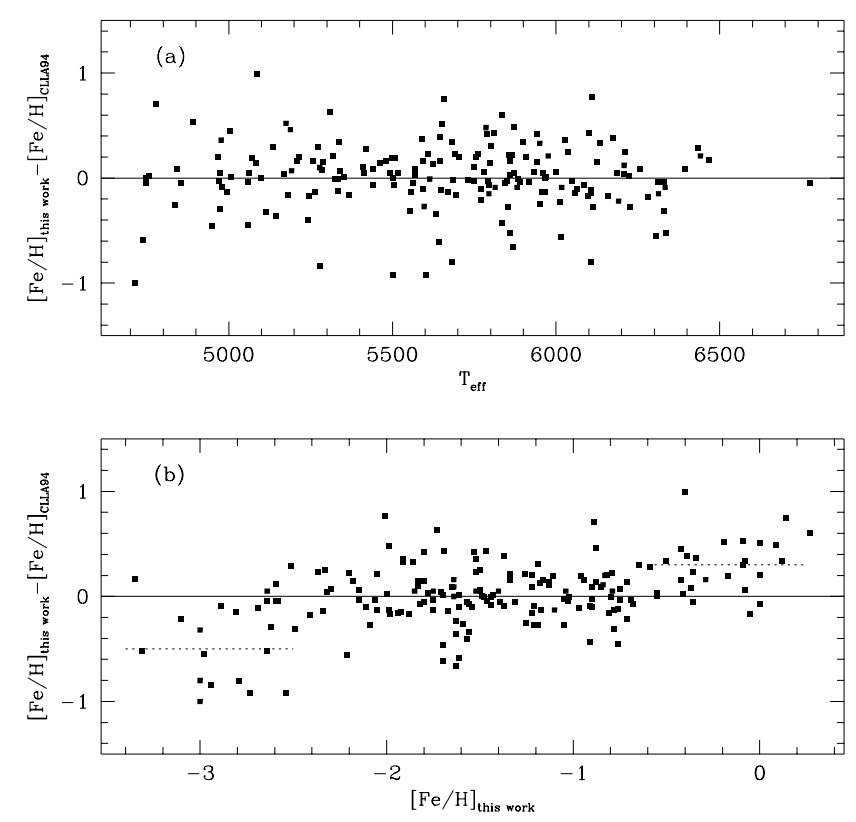

Fig. 8. a) Differences between the metallicities adopted in this work and those derived by Carney et al. (1994) versus effective temperature. b) The same that in a) versus metallicity. No trend may be appreciated with temperature. However, our $[\mathrm{Fe} / \mathrm{H}]$ estimate is 0.5 dex higher for $[\mathrm{Fe} / \mathrm{H}]<-2.5$, and 0.3 dex lower for $[\mathrm{Fe} / \mathrm{H}]>-0.5$ 


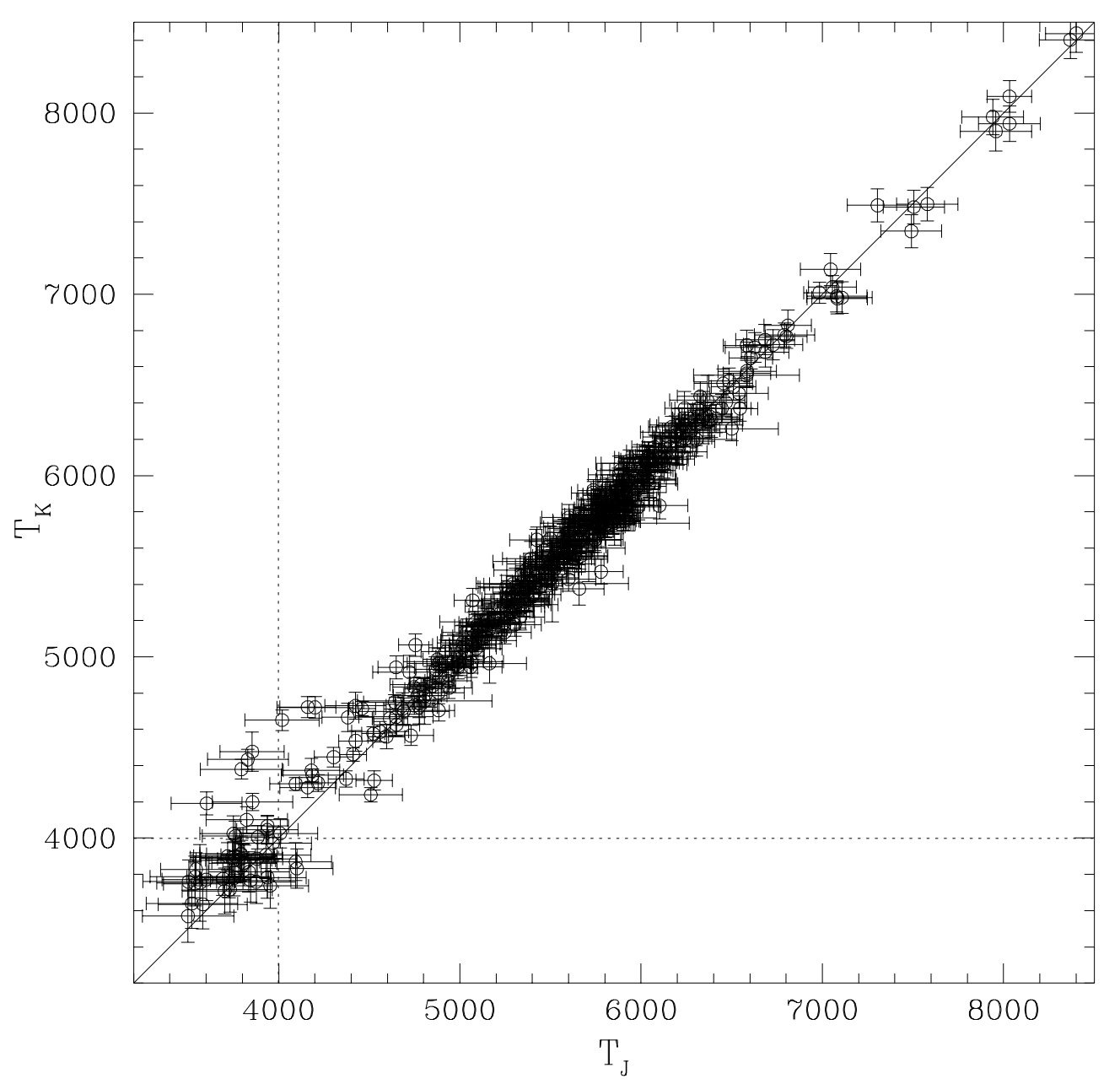

Fig. 9. Comparison between $T_{J}$ and $T_{K}$. The dotted lines mark the lower limit $\left(T_{\text {eff }}=4000 \mathrm{~K}\right)$ where models begin to falter due to the lacking of molecular opacity sources. It is worth notice the progressive departure of the points from the diagonal towards the cooler stars

have been considered for determining $E(B-V)$. The first one is based on the intrinsic relation $(V-K):(J-K)$ (Carney 1983a), considering $\mathrm{E}(V-K)=2.72 E(B-V)$ (Savage \& Mathis 1979). The histogram obtained with all the values within the application range is presented in Fig. 5. The second method is based on the $\beta$ index and Strömgren photometry. Schuster \& Nissen (1989) have calibrated $E(b-y)$ with an accuracy of 0.015 mag. The result of applying this calibration to the stars of the sample is showed in the histogram of Fig. 5.

Moreover a number of stars of the sample have extinction estimations determined by other authors (Laird et al. 1987; Beers et al. 1990) based on reddening maps by Burnstein \& Heiles (1982). The correlation between the different methods is acceptable for $E(B-V)<0.1 \mathrm{mag}$, over this value it worsens.

When Strömgren and $\beta$ photometry was available the method by Schuster \& Nissen (1989) was preferred, considering $E(B-V)=0$ for $E(b-y)<0.025$, and $E(B-$ $V)=1.37 E(b-y)$ otherwise (Crawford 1975). The values taken from the literature, and those determined by means of the intrinsic relation $(V-K):(J-K)$ were considered for the remainder of the stars. Table 4 contains the reddening value assigned to each star in the sample. Note that only a relatively reduced number of stars (about 15 percent) needed reddening correction.

The first line of Table 5 shows the change in temperature induced by $E(B-V)=0.05 \mathrm{mag}$ when applying the IRFM. The effect is stronger for hot stars which emit most of their flux in the visible/UV wavelength range. 


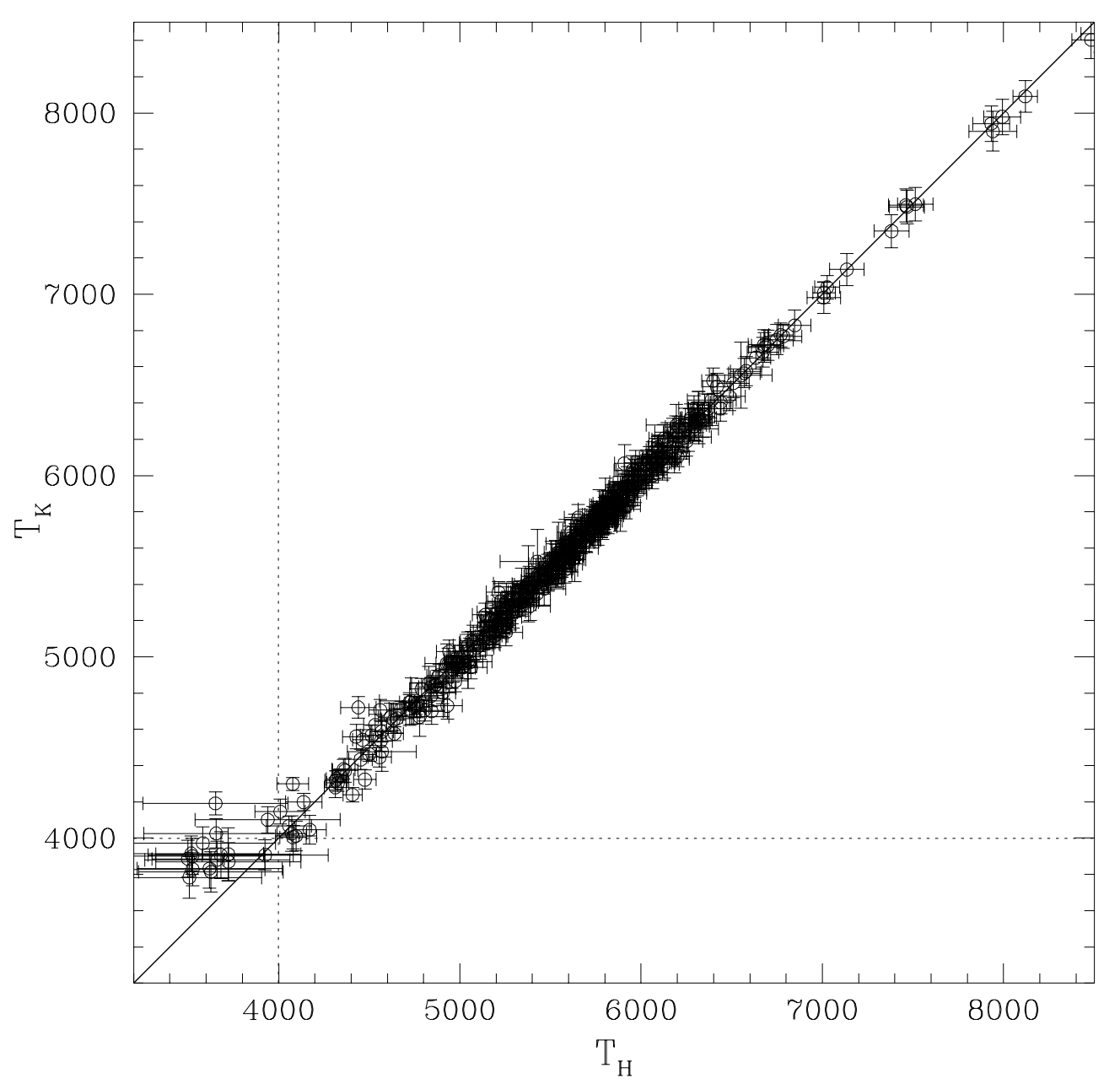

Fig. 10. The same that Fig. 9 for $T_{H}$ and $T_{K}$. Notice the small dispersion around the diagonal line over $4000 \mathrm{~K}$

\subsection{Metallicity and surface gravity}

The effective temperature determination by means of Eq. (4) requires an estimation of the star metallicity and surface gravity. These parameters, however, need not to be very accurate as commented in Sect. 3.3. In particular, it may be concluded that 0.5 dex and 0.3 dex uncertainties in $\log (g)$ and $[\mathrm{Fe} / \mathrm{H}]$, respectively, are sufficient to obtain temperatures within an accuracy of $2 \%$ (see Table 6 ). Therefore, regarding the surface gravity, it is enough to consider an average surface gravity $\log (g)=4.5$ for the subdwarfs. However, taking into account the effect of $T_{\text {eff }}$ on the stellar radius, we have preferred to assign $\log (g)=5$ for the cooler stars, and $\log (g)=4$ for hotest population I stars. These values are compatible with those provided in the reviews by Popper (1980) and Andersen (1991) for dettached binary systems formed by main sequence stars. The gravities adopted for the stars of the sample are listed in Col. 2 of Table 4.
Unfortunately, only a limited number of stars in the sample had their metal abundance determined from fine spectroscopic analysis included in the Catalogue of $[\mathrm{Fe} / \mathrm{H}]$ determinations by Cayrel de Strobel et al. (1992). Therefore for the remainder of stars, photometric metallicity calibrations based on $\delta_{0.6}(U-B)$ index (Carney 1979), and on Strömgren photometry (Schuster \& Nissen 1989), were adopted.

Instead of apply directly these calibrations, we have checked their validity and accuracy. For this purpose, 252 dwarfs and subdwarfs from Cayrel de Strobel et al. (1992) were considered. For each star we assign an averaged abundance giving preference in the weights to recent data based on solid state detectors. We also included, in that group for checking the photometric calibrations, 45 dwarfs from Beers et al. (1990) common to our sample. We have found slight differences between the photometric calibrations and the spectroscopic values which are 

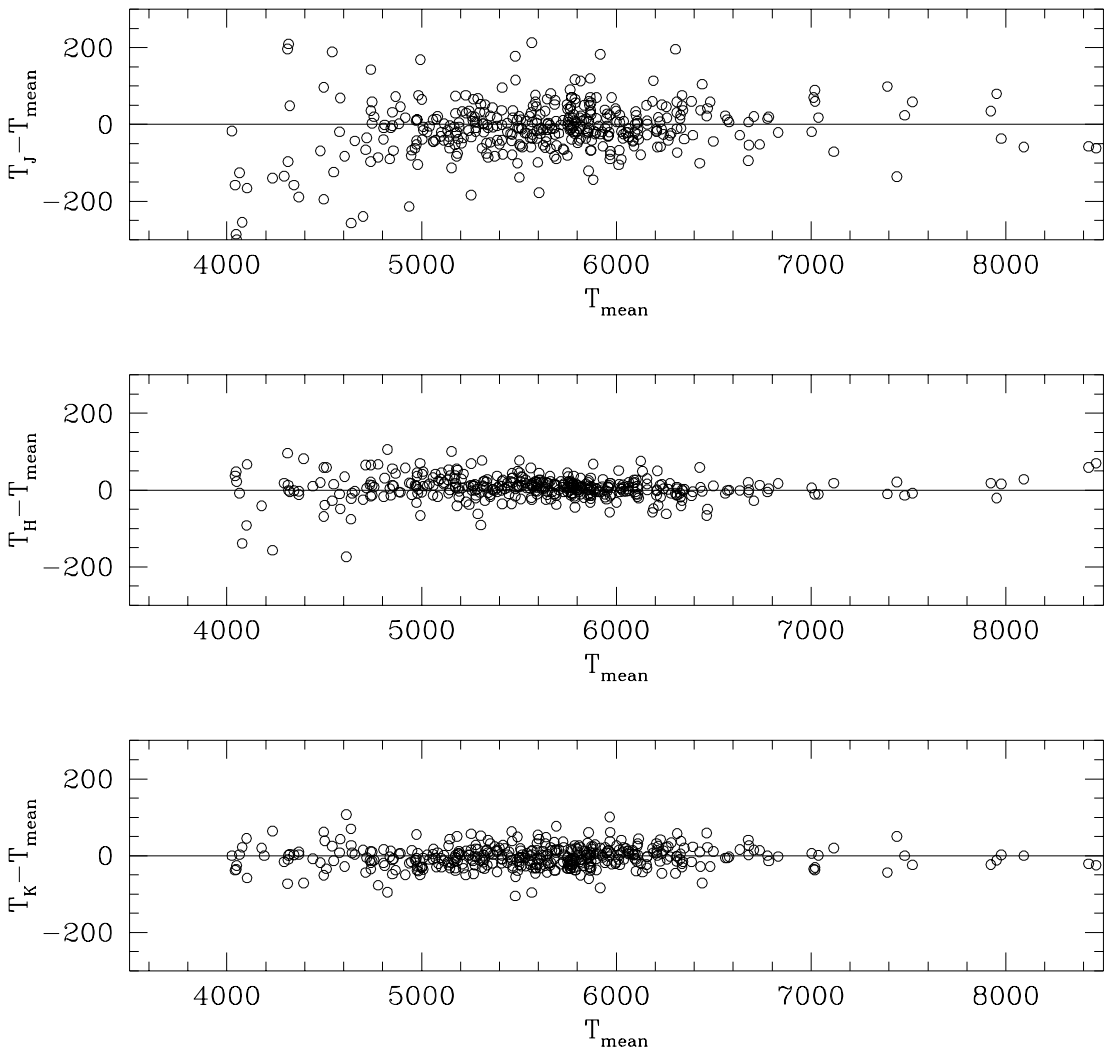

Fig. 11. Differences $T_{J}-T_{\text {mean }}$ (top), $T_{H}-T_{\text {mean }}$ (center) and $T_{K}-T_{\text {mean }}$ (bottom) versus effective temperature adopted. No apparent trend may be appreciated

commented below.

(a) Strömgren photometry calibration.(Schuster \& Nissen 1989): 238 stars with spectroscopic determination of the abundance are within the ranges of the calibrations by Schuster \& Nissen (1989). The following corrections were applied:

$[\mathrm{Fe} / \mathrm{H}]_{\mathrm{phot}}^{\mathrm{F}}=0.82[\mathrm{Fe} / \mathrm{H}]_{\mathrm{spec}}-0.08, \sigma=0.18, \operatorname{dex} n=150$,

$[\mathrm{Fe} / \mathrm{H}]_{\mathrm{phot}}^{\mathrm{G}}=0.93[\mathrm{Fe} / \mathrm{H}]_{\mathrm{spec}}-0.01, \sigma=0.21, \operatorname{dex} n=105$,

where the superindex refers to the calibration of type $\mathrm{F}$ and $\mathrm{G}$ stars. The zero correction is practically negligible, whereas the correction of the slope may imply differences of $0.3-0.5$ dex. If we consider both calibrations altogether, the correction should be:

$[\mathrm{Fe} / \mathrm{H}]_{\text {phot }}=0.85[\mathrm{Fe} / \mathrm{H}]_{\mathrm{spec}}-0.04, \sigma=0.22, \operatorname{dex} n=238$.

This result agrees with the conclusion by Schuster \& Nissen (1989) that their calibrations should perhaps require a slight correction to adjust the revised spectroscopic values (they propose a multiplicative factor of correction for their calibration of 1.15 which roughly coincides with our correction).

(b) Broad band photometry: $\delta_{0.6}(U-B)$ (Carney 1979): 259 stars with spectroscopic determinations are within the range of the calibration of $\delta_{0.6}(U-B)$ index by Carney (1979). This calibration has been extended to the limit permitted by the deblanketing vectors provided by Sandage (1969). Correction differs according to colour.

$$
\begin{gathered}
{[\mathrm{Fe} / \mathrm{H}]_{\mathrm{phot}}=0.83[\mathrm{Fe} / \mathrm{H}]_{\mathrm{spec}}-0.02, \sigma=0.28 \mathrm{dex}, \quad(9)} \\
n=212, \quad(0.35<(B-V) \leq 0.7), \\
{[\mathrm{Fe} / \mathrm{H}]_{\mathrm{phot}}=1.03[\mathrm{Fe} / \mathrm{H}]_{\mathrm{spec}}+0.10, \quad \sigma=0.25 \mathrm{dex}, \quad(10)} \\
n=48, \quad(0.7 \geq(B-V)<1.1),
\end{gathered}
$$

Figure 6 shows the comparison, after corrections, between the spectroscopic and photometric values of the abundance, and the ranges of colour and metallicity covered. Notice the loss of sensitivity, with increasing 

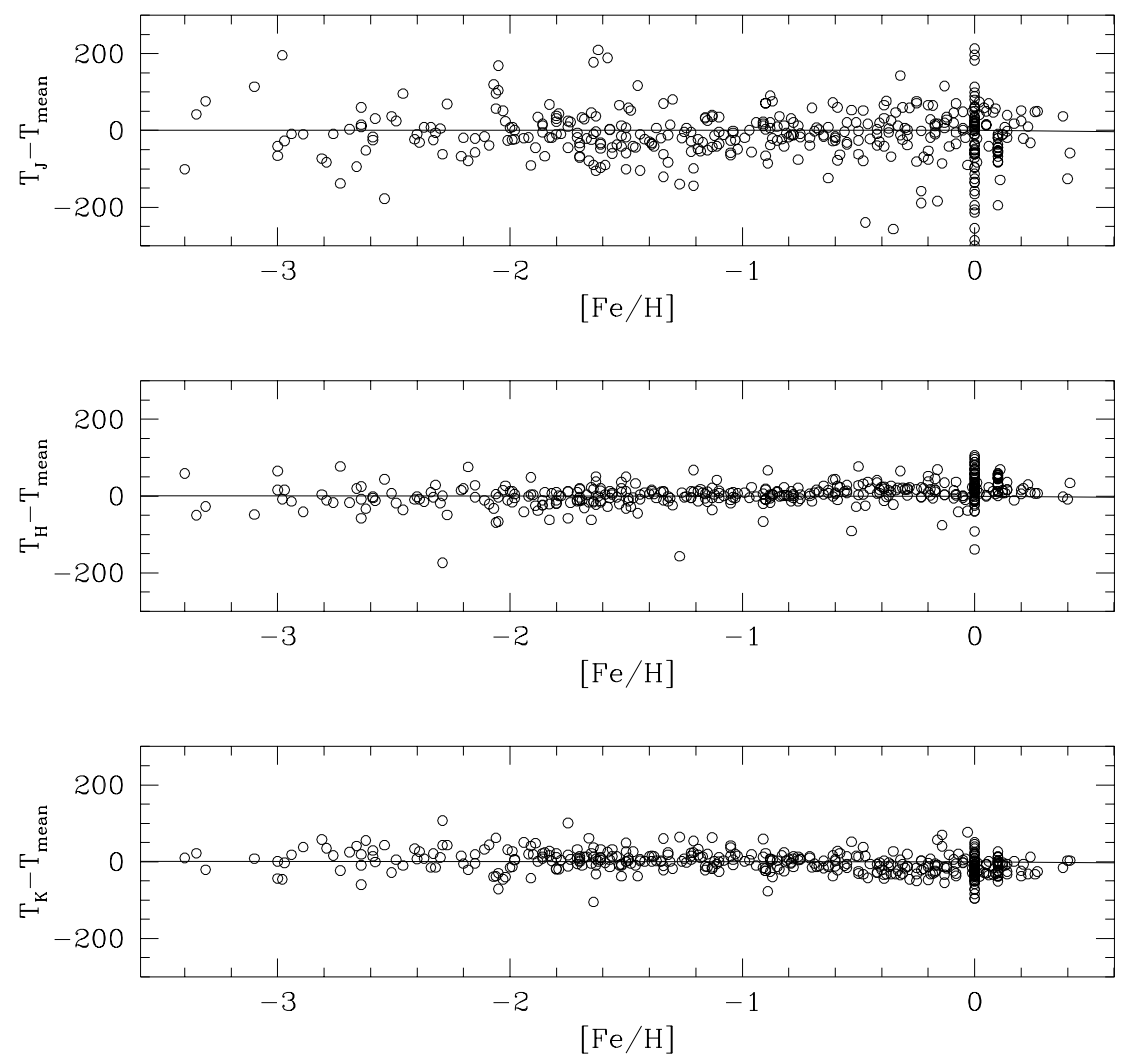

Fig. 12. The same that Fig. 11 versus $[\mathrm{Fe} / \mathrm{H}]$

metallicity, in both calibrations.

In summary, the metallicity has been assigned in a twofold way: Firstly, spectroscopic determined $[\mathrm{Fe} / \mathrm{H}]$ (Cayrel de Strobel et al. 1992), with a mean accuracy within 0.15 dex, have been preferred. Secondly, the revised calibrations by Schuster \& Nissen (1989) (accuracy within $0.2 \mathrm{dex}$ ) and Carney (1979) (accuracy within 0.3 dex) were applied to the stars lacking fine spectroscopic analysis.

For the $\mathrm{M}$ stars extracted from the compilation by Legget (1992), metallicity has been assigned according to their kinematical classification (i.e. $[\mathrm{Fe} / \mathrm{H}]=0$ for young disk stars, $[\mathrm{Fe} / \mathrm{H}]=-1$ for young disk to old disk transition stars, and $[\mathrm{Fe} / \mathrm{H}]=-1.5$ for old disk stars).

The assigned metallicities are listed in Col. 3 of Table 4 .

\subsubsection{Comparison to other determinations}

Our work share 92 and 186 stars in common with the extensive surveys of Ryan \& Norris (1991; R\&N91) and
Carney et al. (1994; CLLA94) respectively. Both works provide a good basis to check the scale of metallicity adopted here.

In Fig. 7, we compare our $[\mathrm{Fe} / \mathrm{H}]$ estimates to the abundances derived by R\&N91 based on a calibration of the $\mathrm{Ca}$ II $\mathrm{H}$ and $\mathrm{K}$ line absorption. The mean difference $[\mathrm{Fe} / \mathrm{H}]_{\text {This work }}-[\mathrm{Fe} / \mathrm{H}]_{\mathrm{R} \& \mathrm{~N} 91}$ is $0.01 \mathrm{dex}$ with $\sigma=0.37$ dex. The scatter of the differences is compatible with the internal errors of both works. No trend of the differences may be appreciated either with temperature (Fig. 7a) or with metallicity (Fig. 7b).

The comparison of the abundances adopted by us with those derived by CLLA94, based on the cross correlation of high resolution low $S / N$ spectra with model templates, shows a fairly good agreement in the range from $[\mathrm{Fe} / \mathrm{H}]=-2.5$ to $[\mathrm{Fe} / \mathrm{H}]=-0.75$. However, noteworthy differences appear for $[\mathrm{Fe} / \mathrm{H}]<-2.5$ and $[\mathrm{Fe} / \mathrm{H}]>-0.5$. The overall mean difference $[\mathrm{Fe} / \mathrm{H}]_{\text {This work }}-[\mathrm{Fe} / \mathrm{H}]_{\mathrm{CLLA} 94}$ is 0.01 dex with $\sigma=0.31$ dex. No trend with temperature may be appreciated in the differences (Fig. 8a), however there exists a clear correlation with metallicity (Fig. 8b). 

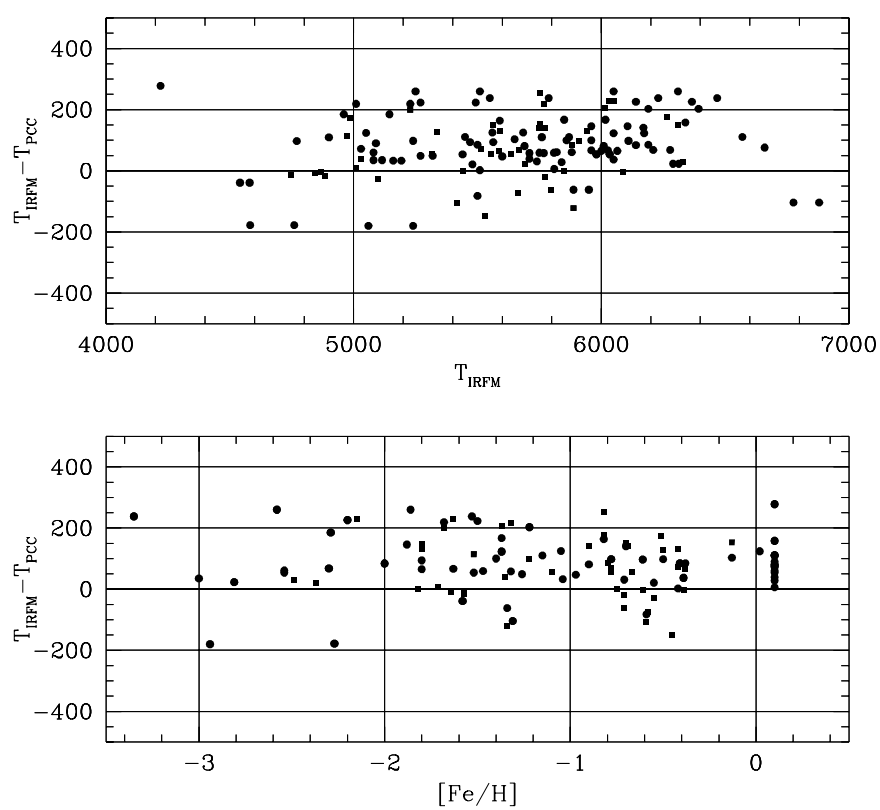

Fig. 13. Differences between the temperatures derived in this work ( $\left.T_{\mathrm{IRFM}}\right)$ and those derived by Peterson \& Carney (1979; Squares), and Carney (1983; Circles). $T_{\mathrm{PCC}}$ are, in average, $100 \mathrm{~K}$ cooler than those derived in the present work. There exists a slight trend with temperature. The differences have a slight slope amounting approximately $100 \mathrm{~K}$ between the extreme points of the overlaping range
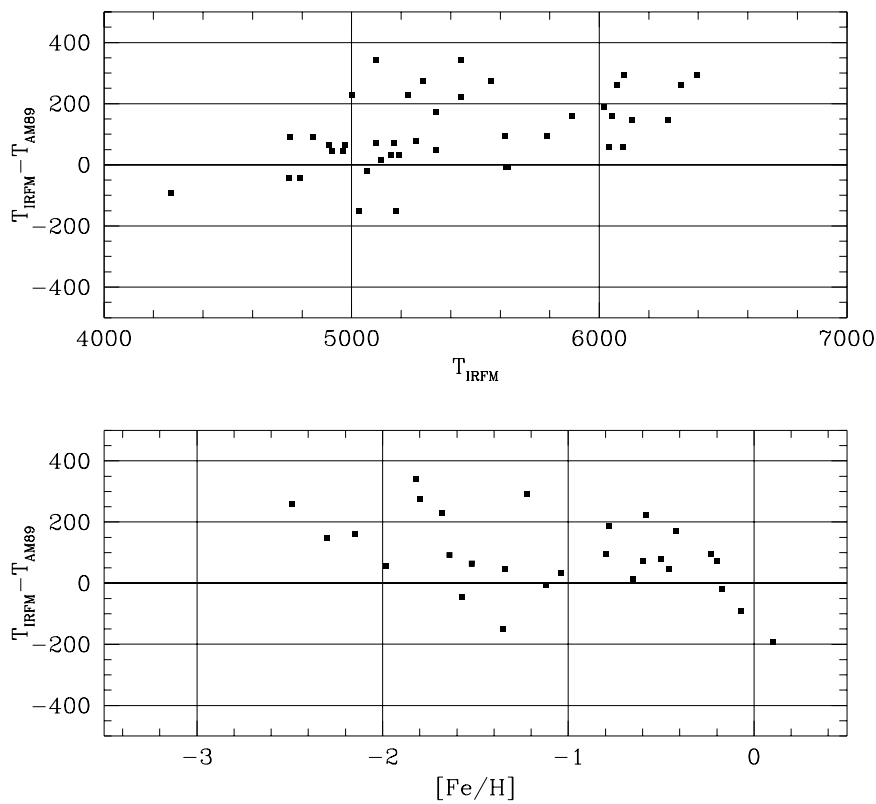

Fig. 14. Differences between the temperatures derived in this work $\left(T_{\mathrm{IRFM}}\right)$ and those derived by Arribas \& Martínez-Roger (1989). $T_{\mathrm{AM} 89}$ are, in average, $150 \mathrm{~K}$ cooler than those derived in the present work. There exist clear trends with temperature and metallicity. The differences may be explained taking into account the different models and absolute flux calibration of the IR flux adopted in both work 
On the one hand, our $[\mathrm{Fe} / \mathrm{H}]$ estimate for the most metal defficient stars $([\mathrm{Fe} / \mathrm{H}]<-2.5)$ is 0.5 dex higher in average than $[\mathrm{Fe} / \mathrm{H}]_{\text {CLLA94, }}$, which implies a difference of $\pm 0.2 \%$ in the temperature derived using the IRFM. On the other, our $[\mathrm{Fe} / \mathrm{H}]$ estimate for stars with $[\mathrm{Fe} / \mathrm{H}]>-0.5$ is 0.3 dex lower in average, which implies a difference of $\pm 0.4 \%$ in temperatures derived using the IRFM.

\section{The temperatures determination}

According to the procedure described in previous sections, we have derived three effective temperatures for each sample star by applying the IRFM in the IR wavelengths considered (Eq. 4). The individual values $T_{J}, T_{H}$ and $T_{K}$, with their errors are listed in Table 4.

Figures 9 and 10 present the comparison between the temperatures obtained in the three different bands taking $T_{K}$ as a reference. The individual errorbars allow to appreciate that the dispersion is compatible with the estimated errors derived from the uncertainties in the input parameters of the IRFM. As expected, the uncertainties are greater for temperatures obtained from $R_{J}$ factors, due to the lesser sensitivity of the IRFM in this band, and the greater photometric error in the measurement of $J$. The consistency of $T_{J}, T_{H}$ and $T_{K}$ is good over 4000 $\mathrm{K}$, however under this temperature there exist noteworthy discrepancies due to the fact that $R_{J}$ and $R_{H^{-}}$-factors lose their sensitivity to temperature in this range.

The final temperature was derived as the mean of $T_{J}$, $T_{H}$ and $T_{K}$ weighted with the inverse of their errors:

$$
\overline{T_{\mathrm{IRFM}}}=\frac{\frac{T_{J}}{\left(\Delta T_{J}\right)}+\frac{T_{H}}{\left(\Delta T_{H}\right)}+\frac{T_{K}}{\left(\Delta T_{K}\right)}}{\left[\frac{1}{\Delta T_{J}}+\frac{1}{\Delta T_{H}}+\frac{1}{\Delta T_{K}}\right]} .
$$

In order to estimate the error of the mean temperature, a linear transmission of the errors was considered, given that the errors in each band are not totally independent:

$$
\Delta T_{\mathrm{IRFM}}=\frac{3}{\left[\frac{1}{\Delta T_{J}}+\frac{1}{\Delta T_{H}}+\frac{1}{\Delta T_{K}}\right]},
$$

where the error in the temperature of each band is defined by

$$
\begin{array}{r}
\left(\Delta T_{i}\right)^{2}=\left[\frac{\Delta T_{i}}{\Delta\left[q\left(\lambda_{i}\right) R\left(\lambda_{i}\right)\right]}\right]^{2}\left(\Delta\left[q\left(\lambda_{i}\right) R\left(\lambda_{i}\right)\right]\right)^{2} \\
+\left[\frac{\Delta T_{i}}{\Delta[\mathrm{Fe} / \mathrm{H}]}\right]^{2}(\Delta[\mathrm{Fe} / \mathrm{H}])^{2} \\
+\left[\frac{\Delta T_{i}}{\Delta \log (g)}\right]^{2}(\Delta \log (g))^{2}
\end{array}
$$

Over $5000 \mathrm{~K}$, the temperatures in the three bands enter the average with similar weight. In that range, the assignation of weights automatically takes into account the unlike sensitivity of the IRFM in the different bands and the individual quality of IR photometry. However, under $5000 \mathrm{~K}$ only $T_{H}$ and $T_{K}$ has been considered in the average, since $R_{J}$ is a very insensitive indicator of temperature for the cooler stars. Under $4000 \mathrm{~K}$, only $T_{K}$ has been considered. This is due to the fact that the coolest models show in the band $H$ a local maximun of flux which is not observed in IR spectra (Lançon \& Rocca-Volmerange 1992). The mean error in the final temperatures is around $1-2 \%$. Notice however, that the uncertainties in the temperatures derived under $4000 \mathrm{~K}$ are greater than the errors determined from Eq. (12) due to the dificulties of models in this range caused by the absence of important sources of opacity associated to some molecules. Likewise, the IRFM is difficult to apply at temperatures over 8000 $\mathrm{K}$ (five stars of the sample have such high temperatures) because as these stars emit a substantial proportion of energy at short wavelengths, the correction for insterstellar extinction and the determination of the bolometric flux are rather uncertain. For these reasons, the temperatures outside the range $4000 \mathrm{~K}<T_{\text {eff }}<8000 \mathrm{~K}$ have a lower level of accuracy (perhaps a most adequated estimation is the duplication of the size of the errorbars quoted in Table 4 for these temperatures).

We show in Figs. 11 and 12 the differences of $T_{J}$, $T_{H}$ and $T_{K}$ from the mean temperature adopted. Over $4000 \mathrm{~K}$ they follow approximately a normal distribution both with effective temperature and metallicity.

\section{Comparison with other determinations}

In this section, we provide the comparison of our temperatures with those derived by other authors for common stars of the sample. Furthermore a detailed analysis of the scale of temperatures derived from the present work will be done in a subsequent paper by considering the mean relations $T_{\text {eff }}:[\mathrm{Fe} / \mathrm{H}]$ and photometric colours $U B V R I J H K$ and $u v b y-\beta$.

The Sun and Procyon, the only dwarfs later than F5 whose diameters has been measured by optical methods, are included in the sample. The agreement between their IRFM temperatures and the direct ones is excellent. In fact, the IRFM provides $6579 \mathrm{~K}$ for Procyon, close to the direct temperature $(6510 \mathrm{~K})$ obtained considering its angular diameter (Hanbury-Brown et al. 1974; Mozurkewich et al. 1991) and its bolometric flux (Code et al. 1976). Regarding the Sun, the value derived from the IRFM determination is $5763 \mathrm{~K}$. This good agreement is a consequence of the procedure followed for scaling the effective temperatures obtained from the IRFM, since the absolute calibration in the near IR was established on the basis of empirical diameters measurements. In addition, it is worthy to notice that the mean temperatures of solar analogue stars (espectral types $\mathrm{G} 0 / 2 \mathrm{~V},[\mathrm{Fe} / \mathrm{H}] \sim 0,(B-V) \sim$ 
$0.63)$ is close to the solar value $(5743 \pm 100 \mathrm{~K}$ vs. 5780 $\mathrm{K}$ for the Sun), and the same for stars of types $\mathrm{F} 3 / 5 \mathrm{~V}$, $[\mathrm{Fe} / \mathrm{H}] \sim-0.05,(B-V) \sim 0.43$ similar in their features to Procyon $(6578 \pm 150 \mathrm{~K}$ vs. $6510 \mathrm{~K})$.

The works by Peterson \& Carney (1979), and Carney (1983a) (hereafter PCC), based on spectrophotometric analysis, contain respectively 43 and 64 stars common to our sample whose range of temperatures and metallicities is similar to that analysed in the present programme. Figure 13 shows the difference $T_{\mathrm{IRFM}}-T_{\mathrm{PCC}}$ versus temperature and metallicity. PCC temperatures are, in average, $100 \mathrm{~K}$ cooler than those derived in the present work. No significant trend of the differences with metallicity may be appreciated. However there could exist a slight slope along the axis of temperatures amounting approximately $100 \mathrm{~K}$ between the extreme points of the overlaping range. This difference is compatible with the uncertainty of the zero point. The different models considered might also explain the observed discrepancies.

There are 18 stars common to the work by Saxner \& Hammarbäck (1985, SH85) devoted to F stars and based on the application of the IRFM. The good agreement is noteworthy as can be appreciated from Table 7 . The differences are compatible with the errors of both works, and the average residuals only amount $5 \pm 63 \mathrm{~K}\left(T_{\mathrm{SH} 85}\right.$ are cooler).

The work by Magain (1987) based on the IRFM presents 11 stars in the metallicity range $(-1,-3)$. The mean difference $T_{\mathrm{IRFM}}-T_{\mathrm{M} 87}$ is $112 \pm 56 \mathrm{~K}$ (Table 8 ) which implies a shift of $1.9 \%$ in the zero point of the temperature scale. This work adopts the IR absolute flux calibration provided by $\mathrm{SH} 85$ which is very close to ours $(+0.3 \%$ in $J$ and $+1.8 \%$ in $K$ ), so the differences of the models considered in each work should account for the shift of the zero.

There are 28 stars common to the work by Arribas y Martínez-Roger (1989, AM89), based on the application of the IRFM using the empirical absolute flux calibration of the IR flux of Vega derived by Mountain et al. (1985), and models by Kurucz (1979) and Gustaffson et al. (1975). The systematic differences found both with temperature and metallicity (Fig. 14) may be explained taking into account that the models used in the present work include new oppacities, and also to the problems of the IR absolute flux calibration derived by Mountain el al. (1985) as described in Paper II.

Bell \& Gustafsson (1989, BG89) present 13 dwarfs common to the present sample. BG89 temperatures are based on the IRFM corrected using IR synthetic colours. Over $4500 \mathrm{~K}$ the differences listed in Table 9 are compatible with a zero point shift of $56 \mathrm{~K}$ ( $T_{\mathrm{BB} 89}$ are hotter). For the coolest stars, where models loses reliability, differences are stronger. The discrepancies are explained taking into account the differences in the bolometric fluxes considered in both works (see Paper III).

Blackwell \& Lynas-Gray (1994, BLG94) have recently applied the IRFM using the new Kurucz's models to a sample of Population I stars. Although only 11 dwarfs are common to the present sample and consequently possible systematic differences are difficult to ascertain, that work is a good source for comparison, given that the main difference respect to the present work is the IR absolute flux calibration. BLG94 adopt the theoretical calibration of the IR flux of Vega provided by Dreiling \& Bell (1980), which differ less than $1 \%$ in all the bands from that adopted here. Table 10 shows the differences. In average, $T_{\mathrm{BLG} 94}$ are $31 \mathrm{~K}$ hotter than ours (the spectroscopic binary HR 219 has been discarded in the mean), this shift in temperature $(\sim 0.6 \%)$ is compatible with the slight difference in the bolometric fluxes (around 1.5\%), as analysed in Paper III.

\section{Summary}

The IRFM has been applied to a sample of approximately 500 main sequence stars later that F0, which cover the metallicity range $(0.5,-3.5)$. Near IR monochromatic fluxes have been used in order to derive $T_{J}, T_{H}$ and $T_{K}$ for each star. The uncertainties of the input parameters needed to apply the IRFM and the induced errors on the three temperatures derived have been computed. The consistency of the temperatures derived in the three different bands is fairly good over $4000 \mathrm{~K}$. The final temperature for each star in the sample has been derived considering the mean of $T_{J}, T_{H}$ and $T_{K}$ weighted with the inverse of their errors. From the analysis of the systematical errors associated to the uncertainty of the absolute flux calibration in the near IR, the expected indetermination of the zero point of the scale of temperatures should be around $1 \%$. However the good agreement between the IRFM and direct temperatures for the Sun and Procyon suggests a lower uncertainty. The mean estimated precision for the final temperatures, considering the effect of both systematical and accidental errors, is around 1.5\%. The comparison with other works shows slight discrepancies which may be explained considering the differences induced by the improvements adopted in the application of the IRFM: The new atmosphere models, the absolute IR flux calibration and the determination of the bolometric fluxes.

Acknowledgements. We are grateful to Dr. R.L. Kurucz for providing us a magnetic tape with his models, and to Prof. B.W. Carney for his remarks and comments concerning several points of this work. We are also grateful to the referee Prof. 
D.E. Blackwell for his comments and suggestions which have certainly improve this paper. This work has been supported by the DGICYT under contracts PB90-0548 and PB91-0526.

\section{References}

Alonso A., Arribas S., Martínez-Roger C., 1994a, A\&A 282, 684 (Paper I)

Alonso A., Arribas S., Martínez-Roger C., 1994b, A\&AS 107, 365 (Paper II)

Alonso A., Arribas S., Martínez-Roger C., 1995, A\&A 297, 197

Anders E., Grevesse N., 1989, Geochim. Cosmochim. Acta 53, 197

Andersen J., 1991, A\&A Rev. 3, 91

Arribas S., Martínez-Roger C., 1987, A\&A 178, 107

Arribas S., Martínez-Roger C., 1988, A\&A 206, 63

Arribas S., Martínez-Roger C., 1989, A\&A 215, 305

Beers T.C., Preston G.W., Shectman S.A., Kage J.A., 1990, AJ 100, 849

Bell R.A., Gustafsson B., 1989, MNRAS 236, 653

Blackwell D.E., Petford A.D., Arribas S., Haddock D.J., Selby M.J., 1990, A\&A 232, 396

Blackwell D.E., Lynas-Gray A.E., Petford A.D., 1991, A\&A 245,567

Blackwell D.E., Petford A.D., 1991, A\&A 250, 459

Blackwell D.E., Lynas-Gray A.E., 1994, A\&A 282, 899

Böhm-Vitense E., 1981, ARA\&A 19, 295

Burnstein D., Heiles C., 1982, AJ 87, 1165

Carney B.W., 1979, ApJ 233, 211

Carney B.W., 1982, AJ 87, 1527

Carney B.W., 1983a, AJ 88, 610

Carney B.W., 1983b, AJ 88, 623

Carney B.W., Latham D.W., 1987, AJ 92, 116

Carney B.W., Latham D.W., Laird J.B., Aguilar L.A., 1994, AJ 107, 2240

Cayrel de Strobel G., Hauck B., François P., Thevénin F. Friel E., Mermilliod M., Borde S., 1992, A\&AS 95, 273

Code A.D., Davis J., Bless R.C., Hanbury-Brown R., 1976, ApJ 203, 417

Crawford D.L., 1975, AJ 80, 955

Dreiling L.A., Bell R.A., 1980, ApJ 241, 736

Glass I.S., 1985, Irish. A. J. 17, 1

Gustafsson B., Bell R.A., Ericksson K., Nordlung Å., 1975, A\&A 42,407

Hanbury-Brown R., Davis J., Allen L.R., 1974, MNRAS 167, 121

Hayes D.S., Latham D.W., 1975, AJ 197, 593
Kidger M., 1992, Carlos Sánchez Telescope Technical note series. Supplement to technical note \# 16. Calibration star programme results

King J.R., 1994, AJ 107, 1165

Kurucz R.L., 1979a, ApJS 40, 1

Kurucz R.L., 1979b, in: Philip A.G. Davis (ed.), Problems of Calibration of Multicolor Photometric Systems, Dudley Obs. Rep. No. 14, p. 363

Kurucz R.L., 1991, Precision photometry: Astrophysics of the Galaxy. In: Davis Philip A.G., Upgren A.R. and Janes K.A. (eds.). L. Davis Press, Schenectady

Kurucz R.L., 1993, Atmosphere models, (Private communication)

Lançon A., Rocca-Volmerange B., 1992, A\&ASS 96, 593

Laird J.B., Carney B.W., Latham D.W., 1987, AJ 95, 1843

Landolt-Börnstein, 1982c, New Series, Gp VI, Vol. 2, Astronomy and Astrophys., Subvolume C. Springer, BerlinHeidelberg-New York

Legget S.K., 1992, ApJS 82, 351

Magain P., 1987, A\&A 181, 323

Manduca A., Bell R.A., 1979, PASP 91, 848

Mountain C.M., 1983, Ph. D. Thesis, Imperial College of Science and Technology, University of London

Mountain C.M., Leggett S.K., Selby M.J., Blackwell D.E., Petford A.D., 1985, A\&A 151, 399

Mozurkewich D., Johnston K.J., Simon R.S., et al., 1991, AJ 101, 2207

Peterson R.C., Carnew B.W., 1979, ApJ 231, 762

Petford A.D., Blackwell D.E., Booth A.J., et al., 1988, A\&A 203, 341

Popper D.M., 1980, ARA\&A 18, 115

Ryan S.G., Norris J.E., AJ 101, 1835

Sandage A., 1969, ApJ 158, 1115

Sandage A., 1983, AJ 88, 1159

Sandage A., Kowal C., 1986, AJ 91, 1140

Savage B.D., Mathis J.S., 1979, ARA\&A 17, 13

Saxner M., Hammarbäck G., 1985, A\&A 151, 372

Schuster W.J., Nissen P.E., 1988, A\&AS 73, 225

Schuster W.J., Nissen P.E., 1989, A\&A 221, 65

Tüg H., White N.M., Lockwood G.W., 1977, A\&A 61, 67

Van Altena W.F., Lee J.T., Hanson R.B., Lutz T.E., 1988, "Calibration of Stellar Ages". In: Davis Philip A.G. (ed.). L. Davis Press, Schenectady, New York, p. 175

Walker R.G., Cohen M., An atlas of selected calibrated stellar spectra. NASA Contractor Report 177604, September 1992. NASA Ames Research Center, Moffett Field, California 94035-1000 
Table 4. The input parameters needed to the application of the IRFM for each star of the sample. Column 1: Identification, the Giclas number has been preferred when available HD, BD, HR and GJ numbers were used otherwise. The stars are ordered in right ascension. Column 2: Surface gravity. Column 3: Metallicity. Column 4: Bolometric flux in ( $\left.\mathrm{erg} \mathrm{cm}^{-2} \mathrm{~s}^{-1}\right)$. Column 5: Interstellar redenning. Column 6: Monochromatic flux in the band $J$ in $\left(10^{2} \mathrm{erg} \mathrm{cm}^{-2} \mathrm{~s}^{-1} \mathrm{~nm}^{-1}\right)$. Column $7: q$-factor in the band $J$. Column 8: Temperature derived in the band $J$ (units are K). Column 9: Error in $T_{J}$ computed considering errors in $F_{\mathrm{Bol}}, F_{J}, \log (g)$ and $[\mathrm{Fe} / \mathrm{H}]$. Columns 10-13: The same that Cols. 6-9 for the band H. Columns 14-17: The same that Cols. 6-9 for the band $K$. Column 18: The weighted mean temperature derived from $T_{J}, T_{H}$ and $T_{K}$. Temperatures in brackets have not been considered in the mean as explained in Sect. 5. Column 19: Mean error computed considering linear transmission of errors from Cols. 9, 13 and 17. Temperatures under $4000 \mathrm{~K}$ are given in parentheses without estimation of the total error because of the uncertainties of the model fluxes in this range

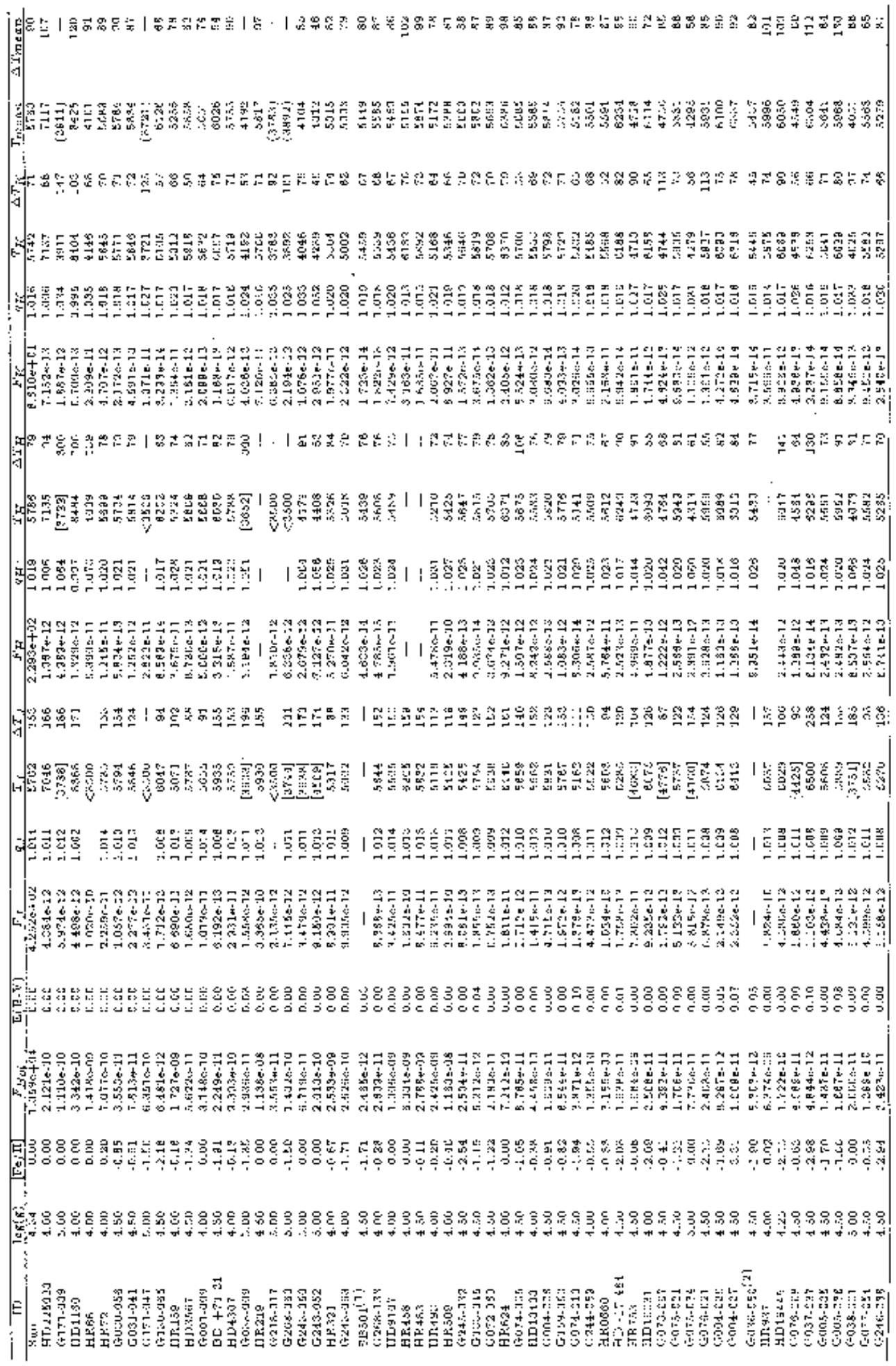


Table 4. continued

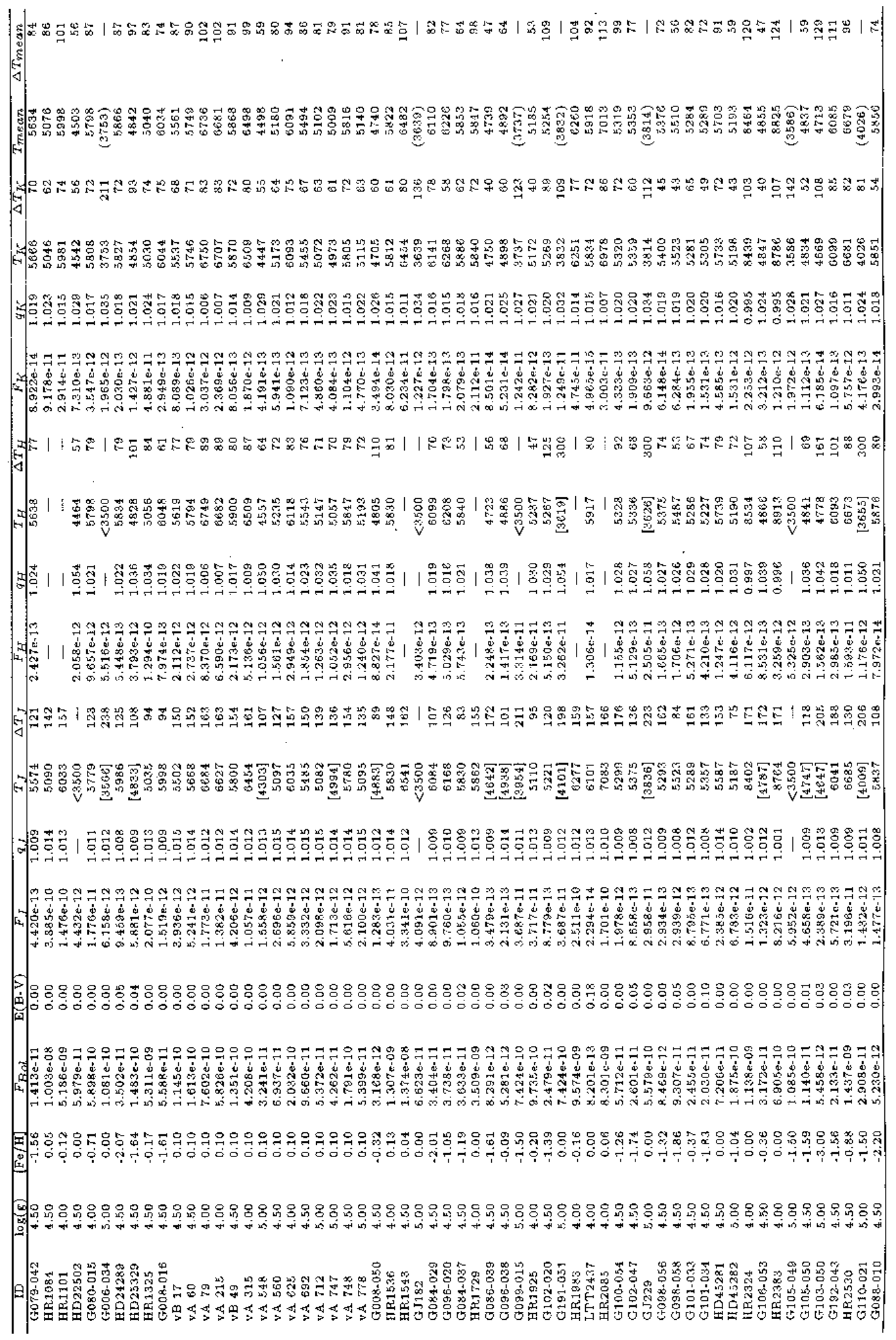


Table 4. continued

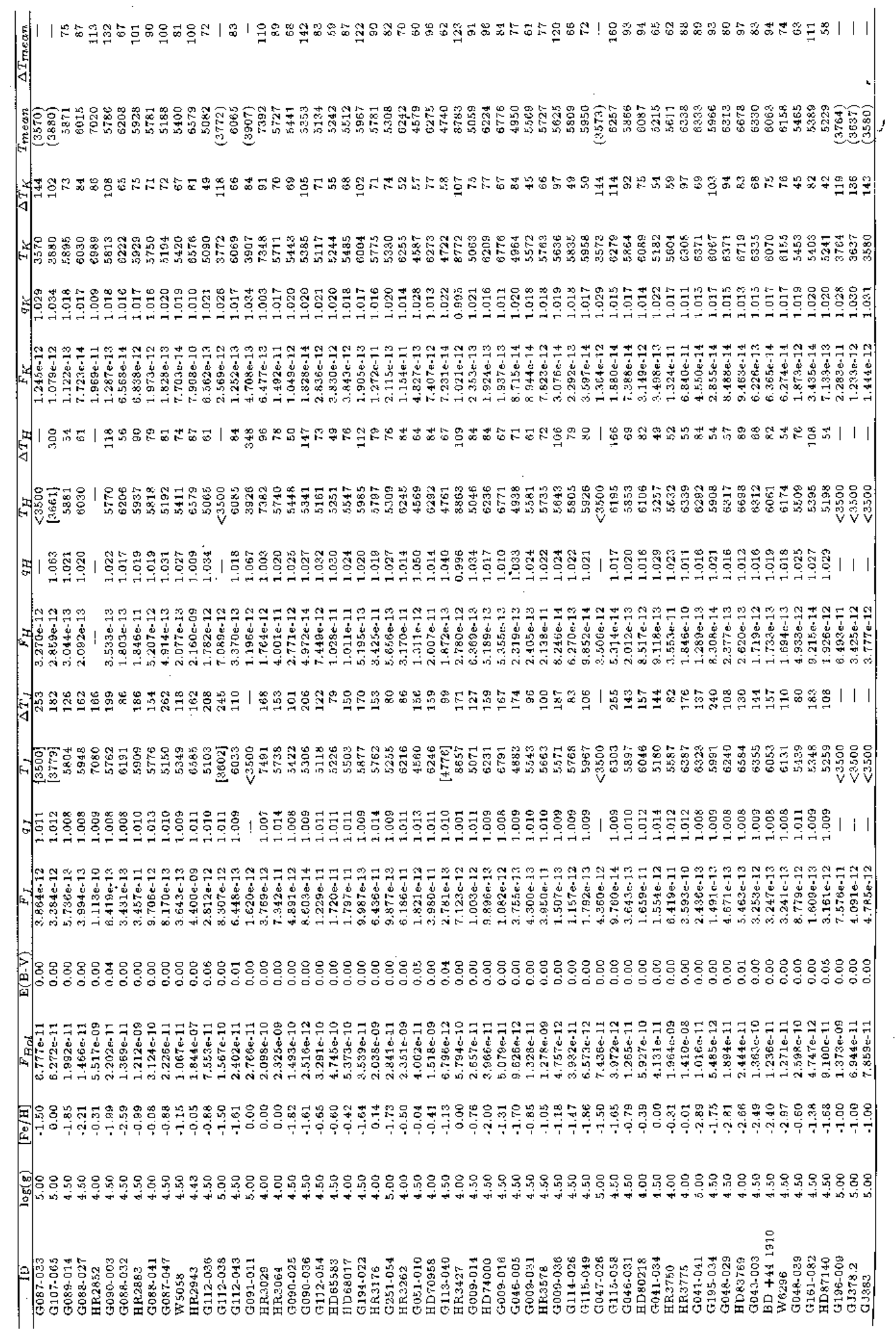


Table 4. continued

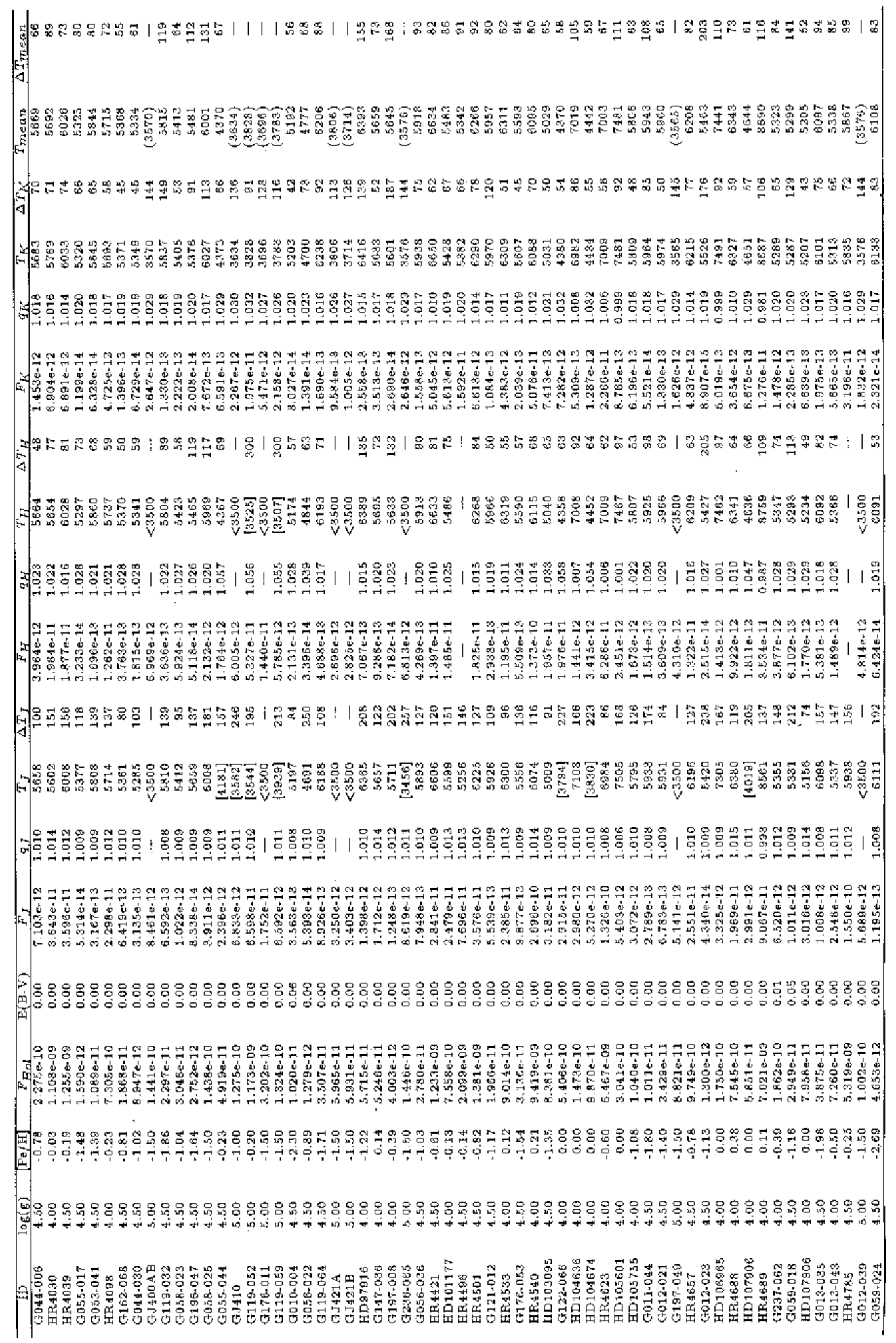


Table 4. continued

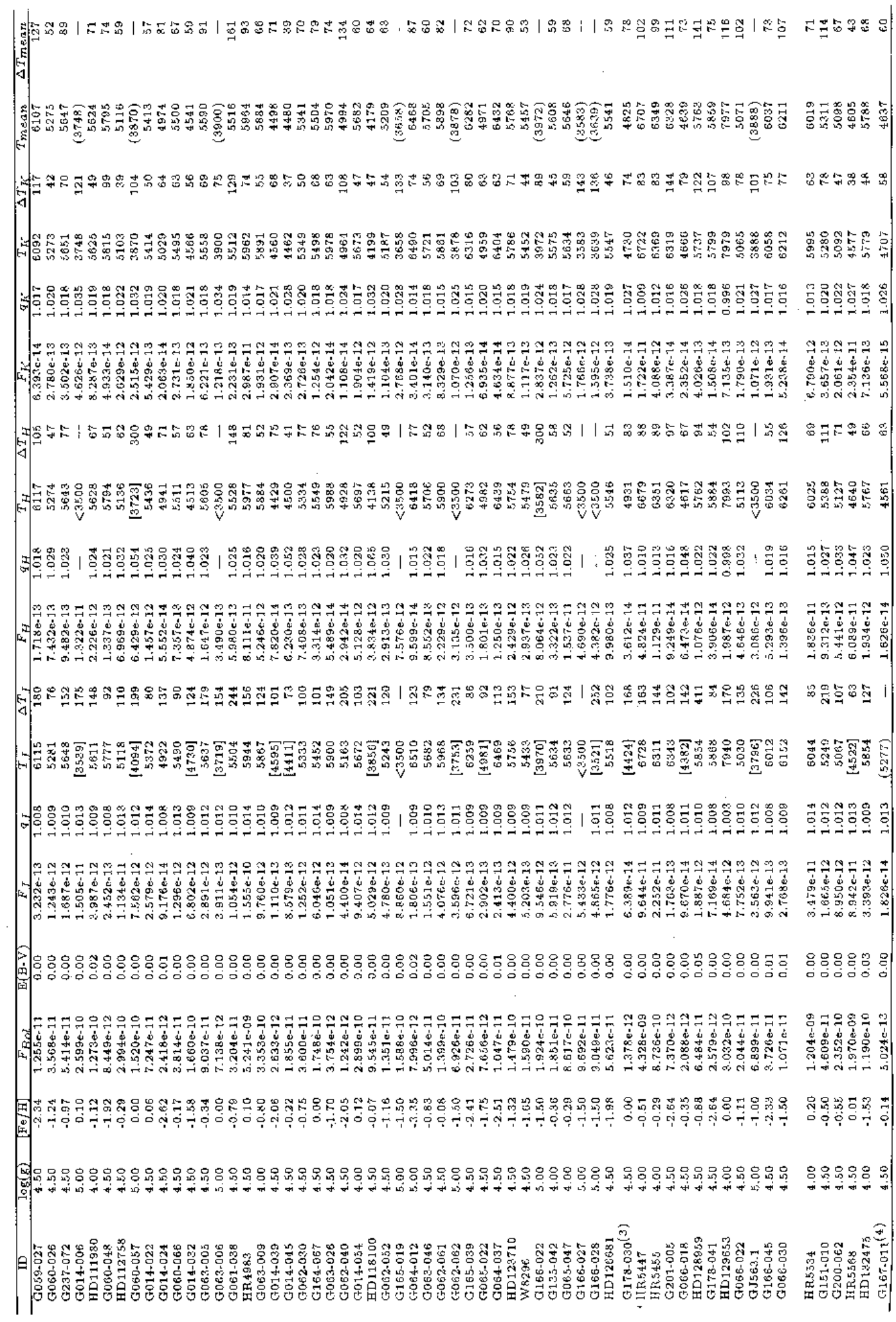


Table 4. continued

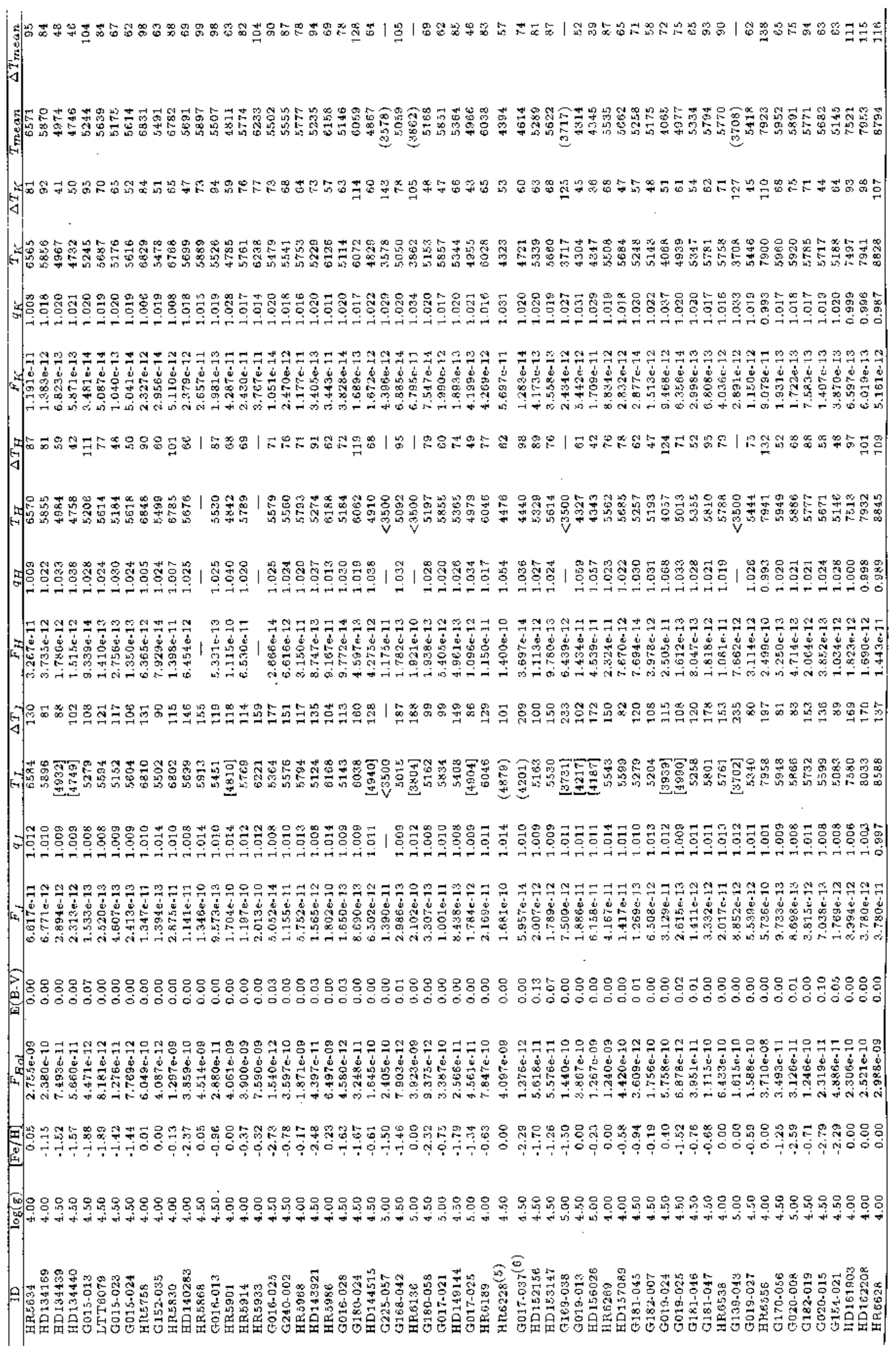


Table 4. continued

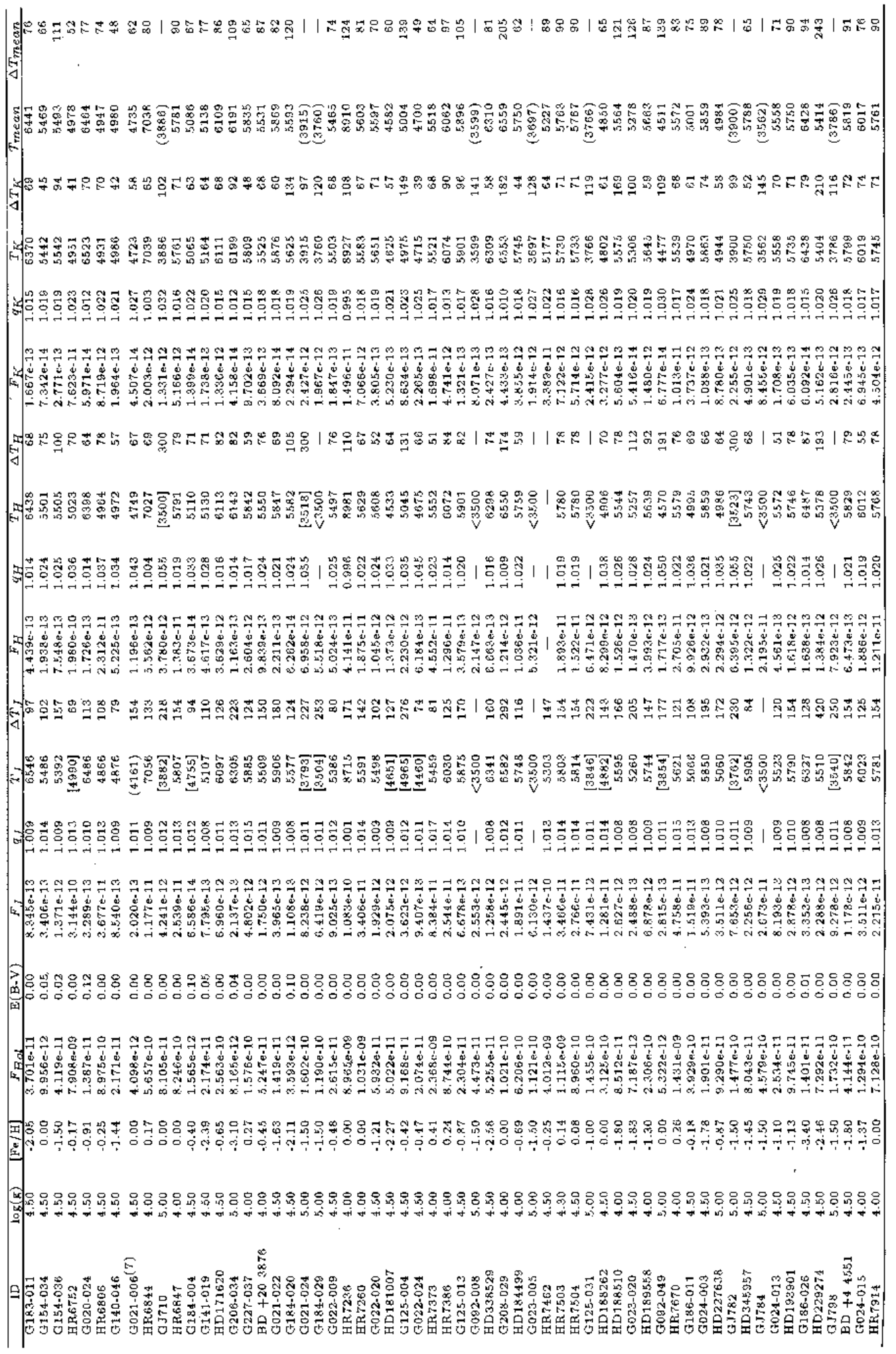


Table 4. continued

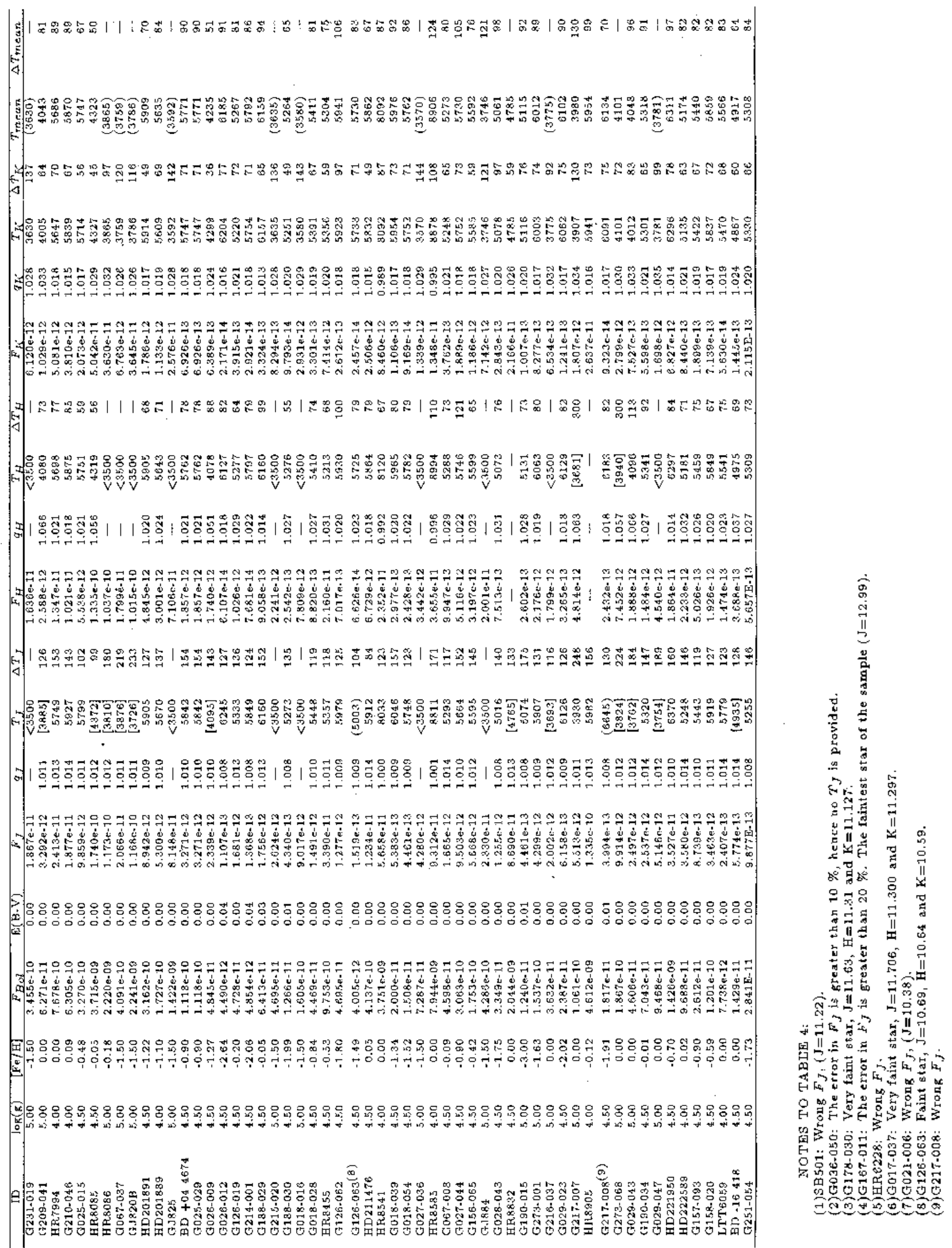

